\title{
THE VANISHING DISCOUNT APPROACH FOR THE AVERAGE CONTINUOUS CONTROL OF PIECEWISE DETERMINISTIC MARKOV PROCESSES
}

\author{
O. L. V. COSTA, ${ }^{*}$ Escola Politécnica da Universidade de São Paulo \\ F. DUFOUR, ${ }^{* *}$ Université Bordeaux I and INRIA Bordeaux Sud Ouest
}

\begin{abstract}
This work is concerned with the existence of an optimal control strategy for the longrun average continuous control problem of piecewise-deterministic Markov processes (PDMPs). In Costa and Dufour (2008), sufficient conditions were derived to ensure the existence of an optimal control by using the vanishing discount approach. These conditions were mainly expressed in terms of the relative difference of the $\alpha$-discount value functions. The main goal of this paper is to derive tractable conditions directly related to the primitive data of the PDMP to ensure the existence of an optimal control. The present work can be seen as a continuation of the results derived in Costa and Dufour (2008). Our main assumptions are written in terms of some integrodifferential inequalities related to the so-called expected growth condition, and geometric convergence of the post-jump location kernel associated to the PDMP. An example based on the capacity expansion problem is presented, illustrating the possible applications of the results developed in the paper.
\end{abstract}

Keywords: Piecewise-deterministic Markov process; continuous time; long-run average cost; optimal control; integro-differential optimality inequation; vanishing discount approach

2000 Mathematics Subject Classification: Primary 60J25; 90C40; 93E20

\section{Introduction}

Piecewise-deterministic Markov processes (PDMPs) have been introduced in the literature by Davis [4], [5] as a general class of stochastic models suitable for formulating optimization problems in queueing and inventory systems, maintenance/replacement models, investment scheduling, and many other areas of operations research. PDMPs are a family of Markov processes involving deterministic trajectories punctuated by random jumps. On a state space $E$ the motion of a PDMP is determined by three parameters: the flow $\phi$, the jump rate $\lambda$, and the transition measure $Q$. Between two jumps, the trajectory of a PDMP follows the flow $\phi$. The jumps occur either spontaneously in a Poisson-like fashion with rate $\lambda$ or when the flow hits the boundary of the state space. In either case the location of the process at the jump time is selected by the transition measure $Q$.

Received 2 October 2008; revision received 18 June 2009.

* Postal address: Departamento de Engenharia de Telecomunicações e Controle, Escola Politécnica da Universidade de São Paulo, CEP: 05508 900, São Paulo, Brazil. Email address: oswaldo@lac.usp.br

** Postal address: Institut Mathématiques de Bordeaux, Université Bordeaux I, 351 cours de la Liberation, 33405

Talence Cedex, France. Email address: dufour@math.u-bordeaux1.fr 
Control of PDMPs has received considerable attention in the literature, and the interested reader may consult the book by Davis [5] and the references therein for a complete view of the general theory related to this class of processes. Costa and Dufour [2] studied the long-run average continuous control problem of PDMPs taking values in a general Borel space. At each point $x$ of the state space, a control variable was chosen from a compact action set $\mathbb{U}(x)$ and applied to the jump parameter $\lambda$ and transition measure $Q$. The goal was to minimize the long-run average cost, which was composed of a running cost and a boundary cost (which is added each time the PDMP touches the boundary). Both costs were assumed to be positive but not necessarily bounded. As far as the authors are aware, this was the first time that this kind of problem was considered in the literature. The approach developed in [2] to study the long-run average control problem of PDMPs was somehow related to the classical analysis of Markov decision processes. In particular, sufficient conditions were derived in [2] to ensure the existence of an optimal control by using the so-called vanishing discount approach. These conditions were mainly expressed in terms of the relative difference $h_{\alpha}(x)=\mathscr{g}_{\mathscr{D}}^{\alpha}(x)-\mathscr{g}_{\mathscr{D}}^{\alpha}\left(x_{0}\right)$ of the $\alpha$-discount value functions $\mathscr{g}_{\mathbb{D}}^{\alpha}$. Roughly speaking, it was shown in [2, Section 8] that if there exists a fixed state $x_{0}$ such that $\alpha \mathscr{G}_{\mathcal{D}}^{\alpha}\left(x_{0}\right)$ is bounded in a neighborhood of $\alpha=0$, and if the relative difference $h_{\alpha}$ satisfies $-K_{h} \leq h_{\alpha}(x) \leq b(x)$ for a nonnegative constant $K_{h}$ and a measurable function $b$, then there exists an optimal control. From a practical point of view, this result is not completely satisfactory due to the fact that these conditions depend on the $\alpha$-discount value function $\mathscr{g}_{\mathbb{D}}^{\alpha}$, which may be difficult to obtain explicitly even for simple examples.

The aim of the present work is to overcome this difficulty by providing tractable conditions that are directly related to the primitive data $(\phi, \lambda, Q)$ of the PDMP to ensure the existence of an optimal control. The problem of finding sufficient conditions based on the primitive data of the process is well studied in the literature of the long-run average control problem for Markov decision processes. We do not attempt to present an exhaustive panorama on this topic, but refer the interested reader to [8], [9], [10], [11], [16], and the references therein for detailed discussions on this problem. In this paper we present some assumptions based on integro-differential inequalities related to positive test functions $g$ and $\bar{r}$ (see Assumption 3.1), and on the geometric convergence of the post-jump location kernel associated to the PDMP (see Assumption 3.2), so that, under these hypotheses, we can show that $\alpha \mathcal{g}_{\mathscr{D}}^{\alpha}\left(x_{0}\right)$ is bounded in a neighborhood of $\alpha=0$ and that the relative difference of the $\alpha$-discount value function $h_{\alpha}$ belongs to a weighted-norm space of functions, labeled $\mathbb{B}_{g}(E)$. Another important difference with respect to [2] is that in the present work $h_{\alpha}$ is not necessarily bounded below by a constant. Indeed, this last property was crucial in [2] to show that there exists a solution to an average cost optimality inequality (ACOI) having this same boundedness property, leading to the existence of an optimal control for the PDMP. Here we can only show that a solution to the ACOI exists but belongs to $\mathbb{B}_{g}(E)$. Consequently, the approach presented in [2] cannot be used in the present context and has to be refined.

The paper is organized in the following way. In Section 2 we introduce some notation, basic assumptions, the problem formulation, and the one-stage optimization operators that will be associated to the ACOI. In Section 3 we introduce several assumptions related to the continuity of the parameters, the expected growth condition, and geometric convergence of the post-jump location of the PDMP. The main results are presented in Section 4, which provides sufficient conditions for the existence of a solution to the ACOI and an optimal control strategy for the long-run average continuous control problem of a PDMP in terms of the parameters defining the process. An example based on the capacity expansion problem is presented in Section 5, 
illustrating the possible applications of the results developed in this paper. For the sake of clarity in exposition, most of the proofs are presented in Appendix A.

\section{Notation, basic assumptions, and problem formulation}

The purpose of this section is to present some standard notation and some basic definitions related to the motion of a controlled PDMP $\{X(t)\}$, as well as the control problem and the one-stage optimization operators associated to the ACOI that we will consider throughout the paper. For further details and properties of a PDMP, we refer the reader to [5]. In Subsection 2.1 , besides presenting some basic notation and assumptions, we define the set of admissible control strategies $\mathcal{U}$ as well as the associated motion of the controlled process, and the long-run average and discounted optimal control problems. In Subsection 2.2 we introduce the sets of discrete-time relaxed and ordinary controls, which will be used in the definition of the onestage optimization operators. Roughly speaking, by relaxed control we mean control functions that are probability measures on the control action space, while by ordinary control we mean control functions that are deterministic functions on the control action space. On the one hand, the advantage of considering relaxed controls is that they form a compact set, thus having many important properties, but, on the other hand, we search for an optimal solution that is an ordinary control. Owing to this, in Subsection 2.3 (Definition 2.1) we present two one-stage optimization operators, namely $\mathcal{R}_{\alpha}$, which minimizes over the set of relaxed controls, and $\mathcal{T}_{\alpha}$, which minimizes over the set of ordinary controls. Under the assumptions presented in Section 3, it turns out that the two operators have the same value and, moreover, that the optimal control can be written in a feedback form, that is, a control that depends (deterministically) only on the state variable $x$ (see Theorem A.2 in Appendix A).

\subsection{Presentation of the control problem}

We start this subsection by presenting some notation that will be used throughout the paper. Let $\mathbb{N}$ denote the set of natural numbers, let $\mathbb{R}$ denote the set of real numbers, let $\mathbb{R}_{+}$denote the set of positive real numbers, and let $\mathbb{R}^{d}$ denote the $d$-dimensional euclidian space. We write $\eta$ as the Lebesgue measure on $\mathbb{R}$. For $X$ a metric space, $\mathscr{B}(X)$ represents the $\sigma$-algebra generated by the open sets of $X$. Let $\mathcal{M}(X)$ denote the set of all finite measures on $(X, \mathcal{B}(X))$, and let $\mathcal{P}(X)$ denote the set of all probability measures on $(X, \mathscr{B}(X))$. Let $X$ and $Y$ be metric spaces. The sets of all Borel measurable functions and all bounded functions from $X$ into $Y$ are denoted by $\mathbb{M}(X ; Y)$ and $\mathbb{B}(X ; Y)$, respectively. Moreover, for notational simplicity, we write $\mathbb{M}(X)$, $\mathbb{B}(X), \mathbb{M}(X)^{+}$, and $\mathbb{B}(X)^{+}$for $\mathbb{M}(X ; \mathbb{R}), \mathbb{B}(X ; \mathbb{R}), \mathbb{M}\left(X ; \mathbb{R}_{+}\right)$, and $\mathbb{B}\left(X ; \mathbb{R}_{+}\right)$, respectively. For $g \in \mathbb{M}(X)$ with $g(x)>0$ for all $x \in X, \mathbb{B}_{g}(X)$ is the set of functions $v \in \mathbb{M}(X)$ such that $\|v(x)\|_{g}=\sup _{x \in X}|v(x)| / g(x)<+\infty$. We denote by $\mathbb{C}(X)$ the set of continuous functions from $X$ to $\mathbb{R}$. For $h \in \mathbb{M}(E), h^{+}$and $h^{-}$respectively denote the positive and negative parts of $h$.

The purpose now is to define the motion of a controlled PDMP as well as the long-run average and discounted optimal control problems. Let $E$ be a Borel subset of $\mathbb{R}^{n}$, let $\partial E$ be its boundary, and let $\bar{E}$ be its closure. A controlled PDMP is determined by its local characteristics $(\phi, \lambda, Q)$, as presented in the sequel. The flow $\phi(x, t)$ is a function $\phi: \mathbb{R}^{n} \times \mathbb{R}_{+} \rightarrow \mathbb{R}^{n}$ continuous in $(x, t)$ and such that $\phi(x, t+s)=\phi(\phi(x, t), s)$. For each $x \in E$, the time the flow takes to reach the boundary starting from $x$ is defined as $t_{*}(x):=\inf \{t>0: \phi(x, t) \in \partial E\}$. For $x \in E$ such that $t_{*}(x)=\infty$ (that is, the flow starting from $x$ never touches the boundary), we set $\phi\left(x, t_{*}(x)\right)=\Delta$, where $\Delta$ is a fixed point on $\partial E$. We define the following space of functions 
absolutely continuous along the flow with limit towards the boundary:

$$
\begin{aligned}
\mathbb{M}^{a c}(E)=\left\{g \in \mathbb{M}(E): g(\phi(x, t)):\left[0, t_{*}(x)\right)\right. & \mapsto \mathbb{R} \text { is absolutely continuous for each } \\
x & \left.\in E \text { and, whenever } t_{*}(x)<\infty, \text { the limit } \lim _{t \rightarrow t_{*}(x)} g(\phi(x, t)) \text { exists }\right\} .
\end{aligned}
$$

For $g \in \mathbb{M}^{a c}(E)$ and $z \in \partial E$ for which there exists $x \in E$ such that $z=\phi\left(x, t_{*}(x)\right)$ where $t_{*}(x)<\infty$, we define $g(z)=\lim _{t \rightarrow t_{*}(x)} g(\phi(x, t))$ (note that the limit exists by assumption). As shown in Lemma 2 of [1], for $g \in \mathbb{M}^{a c}(E)$, there exists a function $\mathcal{X} g \in \mathbb{M}(E)$ such that, for all $x \in E$ and $t \in\left[0, t_{*}(x)\right)$,

$$
g(\phi(x, t))-g(x)=\int_{0}^{t} \chi g(\phi(x, s)) \mathrm{d} s .
$$

The local characteristics $\lambda$ and $Q$ depend on a control action $u \in \mathbb{U}$, where $\mathbb{U}$ is a compact metric space (there is no loss of generality in assuming this property for $\mathbb{U}$-see Remark 2.8 of [2]), in the following way: $\lambda \in \mathbb{M}(\bar{E} \times \mathbb{U})^{+}$and $Q$ is a stochastic kernel on $E$ given $\bar{E} \times \mathbb{U}$. For each $x \in \bar{E}$, we define the subsets $\mathbb{U}(x)$ of $\mathbb{U}$ as the sets of feasible control actions that can be taken when the state process is in $x \in \bar{E}$, that is, the control action that will be applied to $\lambda$ and $Q$ must belong to $\mathbb{U}(x)$. The following assumptions, based on the standard theory of Markov decision processes (see, for example, [10]), will be made throughout the paper.

Assumption 2.1. For all $x \in \bar{E}, \mathbb{U}(x)$ is a compact subspace of $\mathbb{U}$.

Assumption 2.2. The set $K=\{(x, a): x \in \bar{E}, a \in \mathbb{U}(x)\}$ is a Borel subset of $\bar{E} \times \mathbb{U}$.

We now present the definition of an admissible control strategy and the associated motion of the controlled process. A control policy $U$ is a pair of functions $\left(u, u_{\partial}\right) \in \mathbb{M}(\mathbb{N} \times E \times$ $\left.\mathbb{R}_{+} ; \mathbb{U}\right) \times \mathbb{M}(\mathbb{N} \times E ; \mathbb{U})$ satisfying $u(n, x, t) \in \mathbb{U}(\phi(x, t))$ and $u_{\partial}(n, x) \in \mathbb{U}\left(\phi\left(x, t_{*}(x)\right)\right)$ for all $(n, x, t) \in \mathbb{N} \times E \times \mathbb{R}_{+}$. The class of admissible control strategies will be denoted by $u$. Consider the state space $\hat{E}=E \times E \times \mathbb{R}_{+} \times \mathbb{N}$. For a control policy $U=\left(u, u_{\partial}\right)$, let us introduce the following parameters for $\hat{x}=(x, z, s, n) \in \hat{E}$ : the flow $\hat{\phi}(\hat{x}, t)=(\phi(x, t), z, s+t, n)$, the jump rate $\hat{\lambda}^{U}(\hat{x})=\lambda(x, u(n, z, s))$, and the transition measure

$$
\hat{Q}^{U}(\hat{x}, A \times B \times\{0\} \times\{n+1\})= \begin{cases}Q(x, u(n, z, s) ; A \cap B) & \text { if } x \in E, \\ Q\left(x, u_{\partial}(n, z) ; A \cap B\right) & \text { if } x \in \partial E,\end{cases}
$$

for $A$ and $B$ in $\mathcal{B}(E)$. From [5, Section 25], it can be shown that, for any control strategy $U=\left(u, u_{\partial}\right) \in U$, there exists a filtered probability space $\left(\Omega, \mathcal{F},\left\{\mathcal{F}_{t}\right\},\left\{\mathrm{P}_{\hat{x}}^{U}\right\}_{\hat{x} \in \hat{E}}\right)$ such that the PDMP $\left\{\hat{X}^{U}(t)\right\}$ with local characteristics $\left(\hat{\phi}, \hat{\lambda}^{U}, \hat{Q}^{U}\right)$ may be constructed as follows. For notational simplicity, the probability $\mathrm{P}_{\hat{x}_{0}}^{U}$ will be denoted by $\mathrm{P}_{(x, k)}^{U}$ for $\hat{x}_{0}=(x, x, 0, k) \in \hat{E}$. Take a random variable $T_{1}$ such that

$$
\mathrm{P}_{(x, k)}^{U}\left(T_{1}>t\right):= \begin{cases}\mathrm{e}^{-\Lambda^{U}(x, k, t)} & \text { for } t<t_{*}(x), \\ 0 & \text { for } t \geq t_{*}(x),\end{cases}
$$

where, for $x \in E$ and $t \in\left[0, t_{*}(x)\right), \Lambda^{U}(x, k, t):=\int_{0}^{t} \lambda(\phi(x, s), u(k, x, s)) \mathrm{d} s$. If $T_{1}$ is equal to $\infty$ then, for $t \in \mathbb{R}_{+}, \hat{X}^{U}(t)=(\phi(x, t), x, t, k)$. Otherwise, independently select an $\hat{E}$-valued 
random variable (labeled $\hat{X}_{1}^{U}$ ) having distribution

$$
\begin{aligned}
\mathrm{P}_{(x, k)}^{U}\left(\hat{X}_{1}^{U} \in A \times B \times\{0\} \times\{k+1\} \mid \sigma\left\{T_{1}\right\}\right) \\
\quad= \begin{cases}Q\left(\phi\left(x, T_{1}\right), u\left(k, x, T_{1}\right) ; A \cap B\right) & \text { if } \phi\left(x, T_{1}\right) \in E, \\
Q\left(\phi\left(x, T_{1}\right), u_{\partial}(k, x) ; A \cap B\right) & \text { if } \phi\left(x, T_{1}\right) \in \partial E .\end{cases}
\end{aligned}
$$

The trajectory of $\left\{\hat{X}^{U}(t)\right\}$ starting from $(x, x, 0, k)$ for $t \leq T_{1}$ is given by

$$
\hat{X}^{U}(t):= \begin{cases}(\phi(x, t), x, t, k) & \text { for } t<T_{1}, \\ \hat{X}_{1}^{U} & \text { for } t=T_{1} .\end{cases}
$$

Starting from $\hat{X}^{U}\left(T_{1}\right)=\hat{X}_{1}^{U}$, we now select the next inter-jump time $T_{2}-T_{1}$ and post-jump location $\hat{X}^{U}\left(T_{2}\right)=\hat{X}_{2}^{U}$ in a similar way. Let us define the components of the PDMP $\left\{\hat{X}^{U}(t)\right\}$ by $\hat{X}^{U}(t)=(X(t), Z(t), \tau(t), N(t))$. From the previous construction, it is easy to see that $X(t)$ corresponds to the trajectory of the system, $Z(t)$ is the value of $X(t)$ at the last jump time before $t, \tau(t)$ is the time elapsed between the last jump and time $t$, and $N(t)$ is the number of jumps of the process $\{X(t)\}$ at time $t$. As in [5, p. 60], we consider the following assumption to avoid any accumulation point of the jump times.

Assumption 2.3. For any $x \in E, U=\left(u, u_{\partial}\right) \in \mathcal{U}$, and $t \geq 0$, we have

$$
\mathrm{E}_{(x, 0)}^{U}\left[\sum_{i=1}^{\infty} \mathbf{1}_{\left\{T_{i} \leq t\right\}}\right]<\infty .
$$

The cost of our control problem will contain two terms, a running cost $f$ and a boundary cost $r$, satisfying the following properties.

Assumption 2.4. We have $f \in \mathbb{M}(\bar{E} \times \mathbb{U})^{+}$and $r \in \mathbb{M}(\partial E \times \mathbb{U})^{+}$.

Define, for $\alpha \geq 0, t \in \mathbb{R}_{+}$, and $U \in \mathcal{U}$,

$$
\begin{aligned}
J^{\alpha}(U, t)= & \int_{0}^{t} \mathrm{e}^{-\alpha s} f(X(s), u(N(s), Z(s), \tau(s))) \mathrm{d} s \\
& +\int_{0}^{t} \mathrm{e}^{-\alpha s} r\left(X(s-), u_{\partial}(N(s-), Z(s-))\right) \mathrm{d} p^{*}(s),
\end{aligned}
$$

where $p^{*}(t)=\sum_{i=1}^{\infty} \mathbf{1}_{\left\{T_{i} \leq t\right\}} \mathbf{1}_{\left\{X\left(T_{i}-\right) \in \partial E\right\}}$ counts the number of times the process hits the boundary up to time $t$, and, for notational simplicity, set $\boldsymbol{J}(U, t)=\boldsymbol{J}^{0}(U, t)$. The long-run average cost we want to minimize over $\mathcal{U}$ is given by $\mathcal{A}(U, x)=\varlimsup_{t \rightarrow+\infty}(1 / t) \mathrm{E}_{(x, 0)}^{U}[J(U, t)]$, and we set $\mathscr{g}_{\mathcal{A}}(x)=\inf _{U \in \mathcal{U}} \mathcal{A}(U, x)$. For the $\alpha$-discounted case, with $\alpha>0$, the cost we want to minimize is given by $\mathscr{D}^{\alpha}(U, x)=\mathrm{E}_{(x, 0)}^{U}\left[J^{\alpha}(U, \infty)\right]$, and we set $\mathscr{G}_{\mathscr{D}}^{\alpha}(x)=\inf _{U \in \mathcal{U}} \mathscr{D}^{\alpha}(U, x)$.

\subsection{Discrete-time relaxed and ordinary controls}

In this subsection we present the definitions of the discrete-time ordinary and relaxed control sets used in the formulation of the one-stage optimization operators, to be presented in Subsection 2.3.

Consider $\mathbb{C}(\mathbb{U})$ equipped with the topology of uniform convergence and $\mathcal{M}(\mathbb{U})$ equipped with the weak* topology $\sigma(\mathcal{M}(\mathbb{U}), \mathbb{C}(\mathbb{U}))$. For $x \in E$, define $\mathcal{P}_{x}(\mathbb{U})$ as the set of measures 
$\mu \in \mathcal{P}(\mathbb{U})$ satisfying $\mu\left(\mathbb{U}\left(\phi\left(x, t_{*}(x)\right)\right)\right)=1$. Here $\mathcal{P}(\mathbb{U})$ and $\mathcal{P}_{x}(\mathbb{U})$ for $x \in E$ are subsets of $\mathcal{M}(\mathbb{U})$ and are equipped with the relative topology.

Let $\mathcal{V}^{r}$ and $\mathcal{V}^{r}(x)$ for $x \in E$ be the sets of all $\eta$-measurable functions $\mu$ defined on $\mathbb{R}_{+}$with values in $\mathcal{P}(\mathbb{U})$ such that $\mu(t, \mathbb{U})=1 \eta$-almost everywhere and $\mu(t, \mathbb{U}(\phi(x, t)))=1 \eta$-almost everywhere, respectively. It can be shown (see Subsection 3.1 of [2]) that $\mathcal{V}^{r}(x)$ is a compact set of the metric space $\mathcal{V}^{r}$. In this case a sequence $\left(\mu_{n}\right)_{n \in \mathbb{N}}$ in $\mathcal{V}^{r}(x)$ converges to $\mu$ if and only if, for all $g \in L^{1}\left(\mathbb{R}_{+} ; \mathbb{C}(\mathbb{U})\right)$,

$$
\lim _{n \rightarrow \infty} \int_{\mathbb{R}_{+}} \int_{\mathbb{U}(\phi(x, t))} g(t, u) \mu_{n}(t, \mathrm{~d} u) \mathrm{d} t=\int_{\mathbb{R}_{+}} \int_{\mathbb{U}(\phi(x, t))} g(t, u) \mu(t, \mathrm{~d} u) \mathrm{d} t .
$$

The sets of relaxed controls can be defined as follows: $\mathbb{V}^{r}(x)=\mathcal{V}^{r}(x) \times \mathcal{P}_{x}(\mathbb{U})$ for $x \in E$ and $\mathbb{V}^{r}=\mathcal{V}^{r} \times \mathcal{P}(\mathbb{U})$. The set of ordinary controls, denoted by $\mathbb{V}($ or $\mathbb{V}(x)$ for $x \in E)$, is defined as above except that it is composed of deterministic functions instead of probability measures. More specifically, we have $\mathcal{V}(x)=\left\{v \in \mathbb{M}\left(\mathbb{R}_{+}, \mathbb{U}\right): v(t) \in \mathbb{U}(\phi(x, t))\right.$ for all $\left.t \in \mathbb{R}_{+}\right\}$, $\mathbb{V}(x)=\mathcal{V}(x) \times \mathbb{U}\left(\phi\left(x, t_{*}(x)\right)\right)$, and $\mathbb{V}=\mathbb{M}\left(\mathbb{R}_{+}, \mathbb{U}\right) \times \mathbb{U}$. Consequently, the set of ordinary controls is a subset of the set of relaxed controls $\mathbb{V}^{r}$ (or $\mathbb{V}^{r}(x)$ for $x \in E$ ) by identifying any control action $u \in \mathbb{U}$ with the Dirac measure concentrated on $u$. Thus, we can write $\mathbb{V} \subset \mathbb{V}^{r}$ (or $\mathbb{V}(x) \subset \mathbb{V}^{r}(x)$ for $x \in E$ ), and from now on we will consider $\mathbb{V}$ (or $\mathbb{V}(x)$ for $x \in E$ ) to be endowed with the topology generated by $\mathbb{V}^{r}$. The necessity to introduce the class of relaxed controls $\mathbb{V}^{r}$ is justified by the fact that in general there does not exist a topology for which $\mathbb{V}$ and $\mathbb{V}(x)$ are compact sets. However, from the previous construction, it follows that $\mathbb{V}^{r}$ and $\mathbb{V}^{r}(x)$ are compact sets.

As in [10, p. 14], we need the set of feasible state/relaxed control pairs to be a measurable subset of $\mathcal{B}(E) \times \mathscr{B}\left(\mathbb{V}^{r}\right)$, that is, we need the following assumption.

Assumption 2.5. We have $\mathcal{K}:=\left\{(x, \Theta): \Theta \in \mathbb{V}^{r}(x), x \in E\right\} \in \mathscr{B}(E) \times \mathscr{B}\left(\mathbb{V}^{r}\right)$.

A sufficient condition is presented in [2, Proposition 3.3] to ensure that Assumption 2.5 holds.

\subsection{One-stage optimization operators}

In this subsection we present some important operators associated to the ACOI, which we will call one-stage optimization operators (see Definition 2.1, below). We also introduce the set of measurable selectors associated to these minimization problems and, in particular, the one of special interest called ordinary feedback measurable selectors.

We consider the following notation: for $x \in \bar{E}, \mu \in \mathcal{P}(\mathbb{U}), h \in \mathbb{M}(E)^{+}$, and $w \in$ $\mathbb{M}(\bar{E} \times \mathbb{U})^{+}$, set

$$
\begin{aligned}
w(x, \mu) & :=\int_{\mathbb{U}} w(x, u) \mu(\mathrm{d} u), \\
Q h(x, \mu) & :=\int_{\mathbb{U}} \int_{E} h(z) Q(x, u ; \mathrm{d} z) \mu(\mathrm{d} u), \\
\text { and } \lambda Q h(x, \mu) & :=\int_{\mathbb{U}} \lambda(x, u) \int_{E} h(z) Q(x, u ; \mathrm{d} z) \mu(\mathrm{d} u) .
\end{aligned}
$$

The following operators will be associated to the optimality equations of the discrete-time problems that will be presented in the sequel. For $\Theta=\left(\mu, \mu_{\partial}\right) \in \mathbb{V}^{r},(x, A) \in E \times \mathscr{B}(E)$, 
and $\alpha \in \mathbb{R}$, define

$$
\begin{aligned}
\Lambda^{\mu}(x, t):= & \int_{0}^{t} \lambda(\phi(x, s), \mu(s)) \mathrm{d} s \\
G_{\alpha}(x, \Theta ; A):= & \int_{0}^{t_{*}(x)} \mathrm{e}^{-\alpha s-\Lambda^{\mu}(x, s)} \lambda Q \mathbf{1}_{A}(\phi(x, s), \mu(s)) \mathrm{d} s \\
& +\mathrm{e}^{-\alpha t_{*}(x)-\Lambda^{\mu}\left(x, t_{*}(x)\right)} Q\left(\phi\left(x, t_{*}(x)\right), \mu_{\partial} ; A\right) .
\end{aligned}
$$

For $h \in \mathbb{M}(E)^{+}$, we define $G_{\alpha} h(x, \Theta):=\int_{E} h(y) G_{\alpha}(x, \Theta ; \mathrm{d} y)$ and, for $x \in E, \Theta=$ $\left(\mu, \mu_{\partial}\right) \in \mathbb{V}^{r}, v \in \mathbb{M}(E \times \mathbb{U})^{+}, w \in \mathbb{M}(\partial E \times \mathbb{U})^{+}$, and $\alpha \in \mathbb{R}$, set

$$
\begin{aligned}
L_{\alpha} v(x, \Theta) & :=\int_{0}^{t_{*}(x)} \mathrm{e}^{-\alpha s-\Lambda^{\mu}(x, s)} v(\phi(x, s), \mu(s)) \mathrm{d} s, \\
H_{\alpha} w(x, \Theta) & :=\mathrm{e}^{-\alpha t_{*}(x)-\Lambda^{\mu}\left(x, t_{*}(x)\right)} w\left(\phi\left(x, t_{*}(x)\right), \mu_{\partial}\right) .
\end{aligned}
$$

For $h \in \mathbb{M}(E)$,

$$
G_{\alpha} h(x, \Theta)=G_{\alpha} h^{+}(x, \Theta)-G_{\alpha} h^{-}(x, \Theta)
$$

and, for $v \in \mathbb{M}(E \times \mathbb{U})$,

$$
L_{\alpha} v(x, \Theta)=L_{\alpha} v^{+}(x, \Theta)-L_{\alpha} v^{-}(x, \Theta),
$$

provided that the difference has a meaning. It will be useful in the sequel to define the function $\mathcal{L}_{\alpha}(x, \Theta)$ as follows: $\mathcal{L}_{\alpha}(x, \Theta):=L_{\alpha} \mathbf{1}_{E \times \mathbb{U}}(x, \Theta)$. In particular, for $\alpha=0$, we write, for simplicity, $G_{0}=G, L_{0}=L, H_{0}=H$, and $\mathcal{L}_{0}=\mathcal{L}$. Measurability properties of the operators $G_{\alpha}, L_{\alpha}$, and $H_{\alpha}$ are shown in [2, Proposition 3.4].

We now present the definitions of the one-stage optimization operators.

Definition 2.1. Let $\alpha \in \mathbb{R}_{+}, \rho \in \mathbb{R}$, and $h \in \mathbb{M}(E)$. Assume that, for any $x \in E$ and $\Upsilon \in \mathbb{V}(x)$, $-\rho \mathcal{L}_{\alpha}(x, \Upsilon)+L_{\alpha} f(x, \Upsilon)+H_{\alpha} r(x, \Upsilon)+G_{\alpha} h(x, \Upsilon)$ is well defined. The (ordinary) one-stage optimization operator is defined by

$$
\mathcal{T}_{\alpha}(\rho, h)(x)=\inf _{\Upsilon \in \mathbb{V}(x)}\left\{-\rho \mathcal{L}_{\alpha}(x, \Upsilon)+L_{\alpha} f(x, \Upsilon)+H_{\alpha} r(x, \Upsilon)+G_{\alpha} h(x, \Upsilon)\right\}
$$

Assume that, for any $x \in E$ and $\Theta \in \mathbb{V}^{r}(x),-\rho \mathcal{L}_{\alpha}(x, \Theta)+L_{\alpha} f(x, \Theta)+H_{\alpha} r(x, \Theta)+$ $G_{\alpha} h(x, \Theta)$ is well defined. The relaxed one-stage optimization operator is defined by

$$
\mathcal{R}_{\alpha}(\rho, h)(x)=\inf _{\Theta \in \mathbb{V}^{r}(x)}\left\{-\rho \mathcal{L}_{\alpha}(x, \Theta)+L_{\alpha} f(x, \Theta)+H_{\alpha} r(x, \Theta)+G_{\alpha} h(x, \Theta)\right\} .
$$

In particular, for $\alpha=0$, we write, for simplicity, $\mathcal{T}_{0}=\mathcal{T}$ and $\mathcal{R}_{0}=\mathcal{R}$.

The sets of measurable selectors associated to $(\mathbb{U}(x))_{x \in E},(\mathbb{V}(x))_{x \in E}$, and $\left(\mathbb{V}^{r}(x)\right)_{x \in E}$ are defined by

$$
\begin{gathered}
s_{\mathbb{U}}=\{u \in \mathbb{M}(\bar{E}, \mathbb{U}): u(x) \in \mathbb{U}(x) \text { for all } x \in \bar{E},\}, \\
s_{\mathbb{V}}=\left\{\left(\nu, v_{\partial}\right) \in \mathbb{M}(E, \mathbb{V}):\left(v(x), \nu_{\partial}(x)\right) \in \mathbb{V}(x) \text { for all } x \in E\right\}, \\
\text { and } \quad s_{\mathbb{V}^{r}}=\left\{\left(\mu, \mu_{\partial}\right) \in \mathbb{M}\left(E, \mathbb{V}^{r}\right):\left(\mu(x), \mu_{\partial}(x)\right) \in \mathbb{V}^{r}(x) \text { for all } x \in E\right\} .
\end{gathered}
$$

For $\alpha \in \mathbb{R}_{+}, \rho \in \mathbb{R}$, and $v \in \mathbb{M}(E)$, the one-stage optimization problem associated to the operator $\mathcal{T}_{\alpha}(\rho, v)$ or $\mathcal{R}_{\alpha}(\rho, v)$ consists of finding a measurable selector $\Upsilon \in \delta_{\mathbb{V}}$ or, respectively, $\Theta \in \S_{\mathbb{V}^{r}}$ such that, for all $x \in E$,

$$
\mathcal{T}_{\alpha}(\rho, v)(x)=-\rho \mathcal{L}_{\alpha}(x, \Upsilon)+L_{\alpha} f(x, \Upsilon)+H_{\alpha} r(x, \Upsilon)+G_{\alpha} v(x, \Upsilon)
$$


or, respectively,

$$
\mathcal{R}_{\alpha}(\rho, v)(x)=-\rho \mathcal{L}_{\alpha}(x, \Theta)+L_{\alpha} f(x, \Theta)+H_{\alpha} r(x, \Theta)+G_{\alpha} v(x, \Theta) .
$$

We note that there exist two natural mappings from $\delta_{\mathbb{U}}$ to $\delta_{\mathbb{V}}$ and from $\delta_{\mathbb{U}}$ to $\mathcal{U}$ (see Propositions 3.8 and 3.10 of [2] for measurability properties), as presented in the next definitions.

Definition 2.2. For $u \in \mathcal{s}_{\mathbb{U}}$, we define the measurable mapping $u_{\phi}$ of the space $E$ into $\mathbb{V}$ by $u_{\phi}: x \rightarrow\left(u(\phi(x, \cdot)), u\left(\phi\left(x, t_{*}(x)\right)\right)\right)$.

Definition 2.3. For $u \in s_{\mathbb{U}}$, we define the measurable mapping $U_{u_{\phi}}$ of the space $\mathbb{N} \times E \times \mathbb{R}_{+}$ into $\mathbb{U} \times \mathbb{U}$ by $U_{u_{\phi}}:(n, x, t) \rightarrow\left(u(\phi(x, t)), u\left(\phi\left(x, t_{*}(x)\right)\right)\right)$.

Remark 2.1. The measurable selectors of the kind $u_{\phi}$ given in Definition 2.2 will be called ordinary feedback measurable selectors in the class $s_{\mathbb{V}} \subset \delta_{\mathbb{V} r}$, and the control strategies of the kind $U_{u_{\phi}}$ given in Definition 2.3 will be called ordinary feedback control strategies in the class $u$.

\section{Assumptions on the parameters of the PDMP}

As mentioned in the introduction, we want to obtain sufficient conditions for the existence of an optimal control for the long-run average control problem of a PDMP posed in Section 2. This is done by assuming a hypothesis on the parameters of the PDMP, instead of, as in [2], considering assumptions which are based on the $\alpha$-discount value functions $\mathscr{g}_{D}^{\alpha}$. The purpose of this section is to introduce these assumptions. The basic idea is that they will yield tractable conditions that may be easier to check in practice.

The following assumption is related to the so-called expected growth condition (see, for instance, Assumption 3.1 of [9] for the discrete-time case, or Assumption A of [8] for the continuous-time case). It will be used, among other things, to guarantee uniform boundedness of $\alpha g_{D}^{\alpha}(x)$ with respect to $\alpha$ (see Theorem 4.1, below).

Assumption 3.1. There exist $b \geq 0, c>0, \delta>0, M \geq 0, g \in \mathbb{M}^{a c}(E), g \geq 1$, and $\bar{r} \in \mathbb{M}(\partial E), \bar{r}(z) \geq 0$, satisfying, for all $x \in E$,

$$
\begin{gathered}
\sup _{a \in \mathbb{U}(x)}\{X g(x)+\operatorname{cg}(x)-\lambda(x, a)[g(x)-Q g(x, a)]\} \leq b, \\
\sup _{a \in \mathbb{U}(x)}\{f(x, a)\} \leq M g(x),
\end{gathered}
$$

and, for all $x \in E$ with $t_{*}(x)<\infty$,

$$
\begin{gathered}
\sup _{a \in \mathbb{U}\left(\phi\left(x, t_{*}(x)\right)\right)}\left\{\bar{r}\left(\phi\left(x, t_{*}(x)\right)\right)+Q g\left(\phi\left(x, t_{*}(x)\right), a\right)\right\} \leq g\left(\phi\left(x, t_{*}(x)\right)\right), \\
\sup _{a \in \mathbb{U}\left(\phi\left(x, t_{*}(x)\right)\right)}\left\{r\left(\phi\left(x, t_{*}(x)\right), a\right)\right\} \leq \frac{M}{c+\delta} \bar{r}\left(\phi\left(x, t_{*}(x)\right)\right) .
\end{gathered}
$$

In the next assumption note that, for any $u \in \S_{\mathbb{U}}, G\left(\cdot, u_{\phi} ; \cdot\right)$ can be seen as the stochastic kernel associated to the post-jump location of a PDMP. This assumption is related to geometric ergodic properties of the operator $G$ (see, for example, the comments on page 122 of [11] or Lemma 3.3 of [9] for more details on this kind of assumption). This assumption is very important because it will be used in particular to ensure that the relative difference of the $\alpha$-discount value functions, $h_{\alpha}$, defined by $h_{\alpha}(x)=\mathscr{g}_{\mathscr{D}}^{\alpha}(x)-\mathscr{g}_{\mathscr{D}}^{\alpha}\left(x_{0}\right)$, belong to the weighted-norm space of functions $\mathbb{B}_{g}(E)$ (see Theorem 4.2, below). 
Assumption 3.2. For each $u \in \mathbb{S}_{\mathbb{U}}$, there exists a probabilitymeasure $v_{u}$ such that $v_{u}(g)<+\infty$ and

$$
\left|G^{k} h\left(x, u_{\phi}\right)-v_{u}(h)\right| \leq a\|h\|_{g} \kappa^{k} g(x)
$$

for all $h \in \mathbb{B}_{g}(E)$ and $k \in \mathbb{N}$, with $a>0$ and $0<\kappa<1$ independent of $u$.

Assumptions 3.3, 3.4, 3.5, and 3.6, below, were introduced in [2]. They are needed to guarantee some convergence and semi-continuity properties of the one-stage optimization operators, the equality between the operators $\mathcal{T}_{\alpha}$ and $\mathcal{R}_{\alpha}$, and the existence of an ordinary feedback measurable selector (see Subsection A.3 and, in particular, Theorem A.2).

Assumption 3.3. We assume that

(i) for each $x \in E$, the restriction of $\lambda(x, \cdot)$ to $\mathbb{U}(x)$ is continuous,

(ii) there exists $\bar{\lambda} \in \mathbb{M}(\bar{E})^{+}$such that, for all $x \in E$ and $a \in \mathbb{U}(x), \lambda(x, a) \leq \bar{\lambda}(x)$, and for $t \in\left[0, t_{*}(x)\right), \int_{0}^{t} \bar{\lambda}(\phi(x, s)) \mathrm{d} s<\infty$, and if $t_{*}(x)<\infty$ then $\int_{0}^{t_{*}(x)} \bar{\lambda}(\phi(x, s)) \mathrm{d} s<\infty$.

Assumption 3.4. There exists a sequence of measurable functions $\left(f_{j}\right)_{j \in \mathbb{N}}$ in $\mathbb{M}(\bar{E} \times \mathbb{U})^{+}$such that, for all $y \in \bar{E}, f_{j}(y, \cdot) \uparrow f(y, \cdot)$ as $j \rightarrow \infty$ and the restriction of $f_{j}(y, \cdot)$ to $\mathbb{U}(y)$ is continuous. There exists a sequence of measurable functions $\left(r_{j}\right)_{j \in \mathbb{N}}$ in $\mathbb{M}(\partial E \times \mathbb{U})^{+}$such that, for all $z \in \partial E, r_{j}(z, \cdot) \uparrow r(z, \cdot)$ as $j \rightarrow \infty$ and the restriction of $r_{j}(z, \cdot)$ to $\mathbb{U}(z)$ is continuous.

Assumption 3.5. For all $x \in \bar{E}$ and $h \in \mathbb{B}(E)$, the restriction of $Q h(x, \cdot)$ to $\mathbb{U}(x)$ is continuous, and the restriction of $Q g(x, \cdot)$ to $\mathbb{U}(x)$ is continuous.

The next definition will be important to characterize the optimal feedback measurable selector and optimal control strategy for our problem.

Definition 3.1. Consider $w \in \mathbb{M}(E)$ and $h \in \mathbb{B}_{g}(E)$. We define

(D1) $\hat{u}(w, h) \in s_{\mathbb{U}}$ as the measurable selector satisfying

$$
\begin{aligned}
& \inf _{a \in \mathbb{U}(x)}\{f(x, a)-\lambda(x, a)[w(x)-Q h(x, a)]\} \\
& =f(x, \hat{u}(w, h)(x))-\lambda(x, \hat{u}(w, h)(x))[w(x)-Q h(x, \hat{u}(w, h)(x))], \\
& \inf _{a \in \mathbb{U}(z)}\{r(z, a)+Q h(z, a)\}=r(z, \hat{u}(w, h)(z))+\operatorname{Qh}(z, \hat{u}(w, h)(z)),
\end{aligned}
$$

(D2) $\hat{u}_{\phi}(w, h) \in \S_{\mathbb{V}}$ as the measurable selector derived from $\hat{u}(w, h)$ through Definition 2.2.

Note that, from Assumption 3.5 and Lemma 8.3.7 of [11], $Q h(x, \cdot)$ is continuous in $\mathbb{U}(x)$. From this and Assumptions 3.3(i) and 3.4, we find that the existence of $\hat{u}(w, h)$ in (D1) follows from Proposition D.5 of [10].

The final assumption is as follows.

Assumption 3.6. There exist $\underline{\lambda} \in \mathbb{M}(E)^{+}, \bar{f} \in \mathbb{M}(E)^{+}$, and $K_{\lambda} \in \mathbb{R}_{+}$such that

(a) $\lambda(y, a) \geq \underline{\lambda}(y)$ and $f(y, a) \leq \bar{f}(y)$ for all $y \in E$ and $a \in \mathbb{U}(y)$,

(b) $\int_{0}^{t_{*}(x)} \exp \left\{c t-\int_{0}^{t} \underline{\lambda}(\phi(x, s)) \mathrm{d} s\right\} \mathrm{d} t \leq K_{\lambda}$ for all $x \in E$,

(c) $\lim _{t \rightarrow+\infty} \exp \left\{c t-\int_{0}^{t} \underline{\lambda}(\phi(x, s)) \mathrm{d} s\right\}=0$ for all $x \in E$ with $t_{*}(x)=+\infty$,

(d) $\lim _{t \rightarrow+\infty} \exp \left\{-\int_{0}^{t} \underline{\lambda}(\phi(x, s)) \mathrm{d} s\right\} g(\phi(x, t))=0$ for all $x \in E$ with $t_{*}(x)=\infty$,

(e) $\int_{0}^{t_{*}(x)} \exp \left\{-\int_{0}^{t} \underline{\lambda}(\phi(x, s)) \mathrm{d} s\right\} \bar{f}(\phi(x, t)) \mathrm{d} t<\infty$. 
Remark 3.1. Note the following consequences of Assumption 3.6.

(i) Assumption 3.6(c) implies that

$$
G_{\alpha}(x, \Theta ; A)=\int_{0}^{t_{*}(x)} \mathrm{e}^{-\alpha s-\Lambda^{\mu}(x, s)} \lambda Q \mathbf{1}_{A}(\phi(x, s), \mu(s)) \mathrm{d} s
$$

and $H_{\alpha} w(x, \Theta)=0$ for any $x \in E$ with $t_{*}(x)=+\infty, A \in \mathcal{B}(E), \alpha \geq-c, \Theta=$ $\left(\mu, \mu_{\partial}\right) \in \mathbb{V}^{r}(x)$, and $w \in \mathbb{M}(\partial E \times \mathbb{U})$.

(ii) Assumption 3.6(a) and (b) imply that $\mathcal{L}_{\alpha}(x, \Theta) \leq K_{\lambda}$ for any $\alpha \geq-c, x \in E$, and $\Theta \in \mathbb{V}^{r}(x)$.

\section{Main results}

In this section we present the main results of the paper, supposing that the assumptions on the parameters of the PDMP presented in Section 3 are satisfied. Our first main result, Theorem 4.1, consists of showing that there exists a fixed state $x_{0}$ such that $\alpha \mathscr{g}_{\mathscr{D}}^{\alpha}\left(x_{0}\right)$ is bounded in a neighborhood of $\alpha=0$. Note that this property was considered to be an assumption in [2], while in the present paper this is a consequence of the assumptions made in the primitive data of the PDMP. Our second main result, Theorem 4.2, states that the relative difference, $h_{\alpha}(x)=\mathscr{g}_{\mathscr{D}}^{\alpha}(x)-\mathscr{g}_{\mathscr{D}}^{\alpha}\left(x_{0}\right)$, of the $\alpha$-discount value function $\mathscr{\mathscr { F }}_{\mathscr{D}}^{\alpha}$ belongs to $\mathbb{B}_{g}(E)$. As mentioned in the introduction, this is the major difference between our results and those presented in [2], where the stronger hypothesis that $h_{\alpha}(x)$ was bounded below by a constant was assumed. Next, as a consequence of Theorems 4.1 and 4.2, it is shown in Proposition 4.1 that there exists a pair $(\rho, h)$ satisfying the ACOI, $h \geq \mathcal{T}(\rho, h)$, where $\rho \in \mathbb{R}_{+}$and $h \in \mathbb{B}_{g}(E)$. Here we again have some important differences between our results and those obtained in [2] since the hypothesis imposed there, that $h_{\alpha}(x)$ was bounded below by a constant, implied that a solution for the ACOI was also bounded below by a constant. From this, we could easily prove the existence of an optimal control for the average cost control problem of PDMPs. In our case we impose only the condition that $h(x)$ is bounded below by $-C g(x)$ for some $C>0$ and, consequently, the approach developed previously by the authors cannot be used. To overcome this difficulty, we show in Proposition 4.2 that, under the assumptions presented in Section 3 , we can obtain, for $\hat{u} \in \mathcal{s}_{\mathbb{U}}$,

$$
\varlimsup_{t \rightarrow+\infty} \frac{1}{t} \varlimsup_{m \rightarrow \infty} \mathrm{E}_{(x, 0)}^{U_{\hat{u}_{\phi}}}\left[\mathcal{T}_{\alpha}(\rho, h)\left(X\left(t \wedge T_{m}\right)\right)\right] \geq 0 .
$$

This technical result will lead in Theorem 4.3 to the main result of the paper, which is the existence of an optimal ordinary feedback control strategy for the long-run average-cost problem of a PDMP.

In order to prove the first two main theorems, several intermediate and technical results are required. For the sake of clarity in exposition, the proofs of these intermediate results and of these two main theorems are presented in Appendix A.

The following theorem states that, for any discount factor $\alpha$ and any state $x \in E, \alpha \mathscr{g}_{D}^{\alpha}(\cdot)$ is bounded.

Theorem 4.1. For any $\alpha>0$ and $x \in E$,

$$
g_{D}^{\alpha}(x) \leq \frac{M}{c+\alpha} g(x)+\frac{M b}{c \alpha} .
$$

Proof. See Appendix A. 
Now, it is shown that, for any state $y$ fixed in $E$, the difference $g_{D}^{\alpha}(\cdot)-\mathscr{g}_{D}^{\alpha}(y)$ belongs to $\mathbb{B}_{g}(E)$.

Theorem 4.2. For any $\alpha>0, x \in E$, and $y \in E$,

$$
\left|\mathscr{g}_{D}^{\alpha}(x)-\mathscr{g}_{D}^{\alpha}(y)\right| \leq \frac{a M^{\prime}}{1-\kappa}(1+g(y)) g(x) .
$$

Proof. See Appendix A.

The purpose of the next result is to show that, by combining the two previous theorems, there exists a pair $(\rho, h)$ in $\mathbb{R}_{+} \times \mathbb{B}_{g}(E)$ satisfying the optimality inequality $h \geq \mathcal{T}(\rho, h)$. A crucial intermediate result to show this is Theorem A.2, presented in Appendix A for the sake of clarity of exposition, which states that, for any function $h \in \mathbb{B}_{g}(E)$, the one-stage optimization operators $\mathcal{R}_{\alpha}(\rho, h)(x)$ and $\mathcal{T}_{\alpha}(\rho, h)(x)$ are equal, and that there exists an ordinary feedback measurable selector for the one-stage optimization problems associated to these operators. This theorem can be seen as an extension of the results obtained in [2] for the case in which the functions under consideration are not necessarily bounded below, as supposed in [2], but instead belong to $\mathbb{B}_{g}(E)$. It must be pointed out that this generalization is not straightforward.

Proposition 4.1. Set $\rho_{\alpha}=\alpha \mathscr{g}_{D}^{\alpha}\left(x_{0}\right)$ and $h_{\alpha}(\cdot)=\mathscr{g}_{\mathscr{D}}^{\alpha}(\cdot)-\mathscr{g}_{\mathscr{D}}^{\alpha}\left(x_{0}\right)$ for a fixed state $x_{0} \in E$. Then the following statements hold.

(i) There exists a decreasing sequence of positive numbers $\alpha_{k} \downarrow 0$ such that $\rho_{\alpha_{k}} \rightarrow \rho$ and, for all $x \in E, \lim _{k \rightarrow \infty} \alpha_{k} \mathcal{g}_{\mathbb{D}}^{\alpha_{k}}(x)=\rho$.

(ii) $h$ defined by $h=\underline{\lim }_{k \rightarrow \infty} h_{\alpha_{k}}$ belongs to $\mathbb{B}_{g}(E)$ and satisfies, for any $x \in E$,

$$
\begin{aligned}
h(x) & \geq \mathcal{T}(\rho, h)(x) \\
& =-\rho \mathcal{L}\left(x, \hat{u}_{\phi}(x)\right)+L f\left(x, \hat{u}_{\phi}(x)\right)+\operatorname{Hr}\left(x, \hat{u}_{\phi}(x)\right)+G h\left(x, \hat{u}_{\phi}(x)\right),
\end{aligned}
$$

where $\hat{u}=\hat{u}(\mathcal{T}(\rho, h), h)$ and $\hat{u}_{\phi}=\hat{u}_{\phi}(\mathcal{T}(\rho, h), h)$ (see Definition 3.1).

Proof. Item (i) is a straightforward consequence of Theorem 4.1. Now, from Theorem 4.2, we find that there exists a constant $C$ independent of $k$ and $x$ such that $\left|h_{\alpha_{k}}(x)\right| \leq C g(x)$. This implies that $h=\underline{\lim }_{k \rightarrow \infty} h_{\alpha_{k}}$ belongs to $\mathbb{B}_{g}(E)$. Following the same arguments as in the proof of Proposition 8.4 of [2], it can be shown that $h(x) \geq \mathcal{R}(\rho, h)(x)$. Applying Theorem A.2, it follows that

$$
\begin{aligned}
h(x) & \geq \mathcal{T}(\rho, h)(x) \\
& =-\rho \mathcal{L}\left(x, \hat{u}_{\phi}(x)\right)+L f\left(x, \hat{u}_{\phi}(x)\right)+H r\left(x, \hat{u}_{\phi}(x)\right)+G h\left(x, \hat{u}_{\phi}(x)\right),
\end{aligned}
$$

where $\hat{u}$ is defined by $\hat{u}(\mathcal{T}(\rho, h), h)$. This shows item (ii) and completes the proof.

In what follows recall the definition of $U_{\hat{u}_{\phi}}$ in Definition 2.3. Next we need to derive a technical result, which is

$$
\varlimsup_{t \rightarrow+\infty} \frac{1}{t} \varlimsup_{m \rightarrow \infty} \mathrm{E}_{(x, 0)}^{U_{\hat{u}_{\phi}}}\left[\mathcal{T}(\rho, h)\left(X\left(t \wedge T_{m}\right)\right)\right] \geq 0,
$$

in order to obtain the existence of an optimal control for the PDMP (this would be easily obtained if $h$ was bounded from below). Proposition 4.2, below, provides this result, but before stating and proving this proposition we need the following lemma. 
Lemma 4.1. Consider an arbitrary $u \in \S_{\mathbb{U}}$, and let $u_{\phi}$ and $U_{u_{\phi}}$ be as in Definitions 2.2 and 2.3 , respectively. For all $x \in E$, define $\hat{g}(x)=-b \mathcal{L}_{-c}\left(x, u_{\phi}(x)\right)+G_{-c} g\left(x, u_{\phi}(x)\right)$. Then $\hat{g} \in \mathbb{B}_{g}(E)$ and $U_{u_{\phi}}$ satisfies

$$
\mathrm{E}_{(x, 0)}^{U_{u_{\phi}}}\left[\hat{g}\left(X\left(t \wedge T_{m}\right)\right)\right] \leq \mathrm{e}^{-c t} g(x)+\frac{b}{c}\left(1-\mathrm{e}^{-c t}\right)+a\|\hat{g}\|_{g} g(x) \kappa^{m}+\|\hat{g}\|_{g} v_{u}(g)+b K_{\lambda} .
$$

Proof. From Corollary A.1 with $\alpha=-c$ and recalling that $\bar{r}(z) \geq 0$, we obtain

$$
-b \mathcal{L}_{-c}\left(x, u_{\phi}(x)\right)+G_{-c} g\left(x, u_{\phi}(x)\right) \leq g(x) .
$$

Clearly, $\hat{g} \in \mathbb{M}(E)$ is bounded from below by $-b K_{\lambda}$ from Assumption 3.6(b) and, thus, $\hat{g} \in \mathbb{B}_{g}(E)$. Since $\hat{g} \in \mathbb{M}(E)$ is bounded from below, it is easy to show that

$$
-b \mathrm{E}_{(x, 0)}^{U_{u_{\phi}}}\left[\int_{0}^{t \wedge T_{m}} \mathrm{e}^{c s} \mathrm{~d} s\right]+\mathrm{E}_{(x, 0)}^{U_{u_{\phi}}}\left[\mathrm{e}^{c\left(t \wedge T_{m}\right)} \hat{g}\left(X\left(t \wedge T_{m}\right)\right)\right] \leq g(x),
$$

by using the same arguments as in the proof of Proposition 4.4 of [2]. Combining Fatou's lemma and Assumption 2.3, we obtain

$$
\mathrm{E}_{(x, 0)}^{U_{u_{\phi}}}[\hat{g}(X(t))] \leq \mathrm{e}^{-c t} g(x)+\frac{b}{c}\left(1-\mathrm{e}^{-c t}\right) .
$$

Clearly, we have

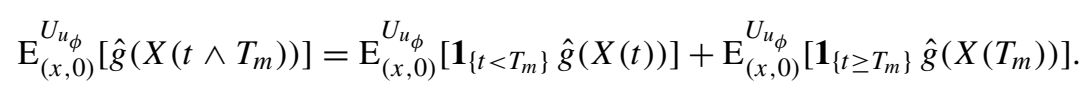

Consequently, we obtain

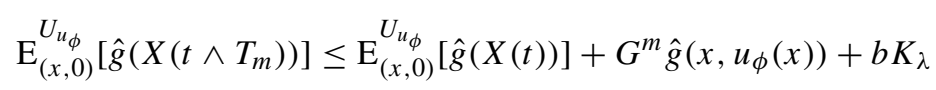

by recalling that $\hat{g}$ is bounded from below by $-b K_{\lambda}$. The result follows from Assumption 3.2 and (4.2).

As a consequence of Lemma 4.1, we obtain the following result.

Proposition 4.2. For all $x \in E, \mathrm{E}_{(x, 0)}^{U_{\hat{u}_{\phi}}}\left[\mathcal{T}(\rho, h)\left(X\left(t \wedge T_{m}\right)\right)\right]$ is well defined and satisfies

$$
\varlimsup_{t \rightarrow+\infty} \frac{1}{t} \varlimsup_{m \rightarrow \infty} \mathrm{E}_{(x, 0)}^{U_{\hat{u}_{\phi}}}\left[\mathcal{T}(\rho, h)\left(X\left(t \wedge T_{m}\right)\right)\right] \geq 0 .
$$

Proof. By definition we have $\mathcal{T}(\rho, h)(x) \geq-\rho \mathcal{L}\left(x, \hat{u}_{\phi}(x)\right)+G h\left(x, \hat{u}_{\phi}(x)\right)$. Therefore, using the definition of $\hat{g}$ in Lemma 4.1 with $u=\hat{u}$, we obtain

$$
\mathcal{T}(\rho, h)(x) \geq-\left(\rho+b\|h\|_{g}\right) K_{\lambda}-\|h\|_{g} \hat{g}(x) .
$$

Consequently, combining Lemma 4.1 and (4.3), we obtain the result.

The next theorem, which is the main result of this paper, shows that the ordinary feedback control $U_{\hat{u}_{\phi}}$ is an optimal strategy for the long-run average-cost problem of a PDMP. 
Theorem 4.3. For all $x \in E, \rho=\mathscr{g}_{\mathcal{A}}(x)=\mathcal{A}\left(U_{\hat{u}_{\phi}}, x\right)$.

Proof. Define

$$
\begin{aligned}
J_{m}^{U_{\hat{u}_{\phi}}}(t, x)=\mathrm{E}_{(x, 0)}^{U_{\hat{u}_{\phi}}}[ & \int_{0}^{t \wedge T_{m}}(f(X(s), \hat{u}(X(s)))-\rho) \mathrm{d} s \\
& \left.+\int_{0}^{t \wedge T_{m}} r\left(X(s-), \hat{u}_{\partial}(X(s-))\right) \mathrm{d} p^{*}(s)+\mathcal{T}(\rho, h)\left(X\left(t \wedge T_{m}\right)\right)\right] .
\end{aligned}
$$

From Proposition 4.2 we know that $\mathrm{E}_{(x, 0)}^{U_{\hat{u}_{\phi}}}\left[\mathcal{T}(\rho, h)\left(X\left(t \wedge T_{m}\right)\right)\right]$ is well defined. Consequently, using Proposition 4.1, we can show that $J_{m} U_{\hat{u}_{\phi}}(t, x) \leq h(x)$ for all $m \in \mathbb{N}$ and $(t, x) \in \mathbb{R}_{+} \times E$. Therefore,

$$
\begin{aligned}
& \mathrm{E}_{(x, 0)}^{U_{\hat{u}_{\phi}}}\left[\int_{0}^{t \wedge T_{m}}(f(X(s), \hat{u}(X(s)))) \mathrm{d} s+\int_{0}^{t \wedge T_{m}} r\left(X(s-), \hat{u}_{\partial}(X(s-))\right) \mathrm{d} p^{*}(s)\right] \\
& +\mathrm{E}_{(x, 0)}^{U_{\hat{u}_{\phi}}}\left[\mathcal{T}(\rho, h)\left(X\left(t \wedge T_{m}\right)\right)\right] \\
& \quad \leq \rho t+h(x) .
\end{aligned}
$$

Now, using Proposition 4.2, it follows that

$$
\begin{aligned}
& \varlimsup_{t \rightarrow+\infty} \frac{1}{t} \varlimsup_{m \rightarrow \infty} \mathrm{E}_{(x, 0)}^{U_{\hat{u}_{\phi}}}\left[\int_{0}^{t \wedge T_{m}}(f(X(s), \hat{u}(X(s)))) \mathrm{d} s\right. \\
&\left.+\int_{0}^{t \wedge T_{m}} r\left(X(s-), \hat{u}_{\partial}(X(s-))\right) \mathrm{d} p^{*}(s)\right] \\
& \leq \rho \varlimsup_{t \rightarrow+\infty} \frac{1}{t} \varlimsup_{m \rightarrow \infty}\left[t \wedge T_{m}\right] .
\end{aligned}
$$

Combining Assumption 2.3 and the monotone convergence theorem, we have

$$
\varlimsup_{t \rightarrow \infty} \frac{1}{t} \mathrm{E}_{(x, 0)}^{U_{\hat{u}_{\phi}}}\left[\int_{0}^{t}(f(X(s), \hat{u}(X(s)))) \mathrm{d} s+\int_{0}^{t} r\left(X(s-), \hat{u}_{\partial}(X(s-))\right) \mathrm{d} p^{*}(s)\right] \leq \rho,
$$

showing that $\mathscr{F}_{\mathcal{A}}(x) \leq \mathcal{A}\left(U_{\hat{u}_{\phi}}, x\right) \leq \rho$. From Theorem 1 of [15, Chapter 5] we easily obtain the reverse inequality, completing the proof.

\section{Example}

In this section we present an example that verifies the assumptions of the paper, so that the results obtained in Section 4 could be applied. It is based on the capacity expansion model, analyzed in [6], [5, Example (34.45)], and by the authors from the stability point of view in [3] and [7]. Capacity expansion models have been introduced in the literature as a general class of stochastic models suitable for formulating the process of adding facilities of similar type over time to meet a rising demand for their services. Typical examples are electrical power generating stations, water resource facilities, computer and communication systems, and large manufacturing facilities. The interested reader may consult [6] and [12] for a survey on capacity expansion, including theoretical results and applications.

The demand for some utility is modeled as a random point process, i.e. it increases by one unit at random times. This demand is met by consecutive construction of identical expansion 
projects. The intensity $\lambda$ of the point process is supposed to be the controlled variable and can assume values in a compact set $\left[\lambda_{a}, \lambda_{b}\right]$ with $\lambda_{b} \geq \lambda_{a}>0$. The construction of a new project is done at a rate $\gamma$ per unit of time and it is completed after the cumulative investment in the current project reaches a value $\tau$. Under completion, the present level of demand is reduced by $\kappa$ units. We will consider for simplicity in this example that $\kappa=1, \gamma=1$, and that $\tau$ does not depend on the present level of demand. Moreover, we suppose that no construction will take place whenever there is no demand and we say, in this case, that the system is in a standby situation. In this case it is assumed for simplicity that a new demand will occur according to a point process with a fixed uncontrolled rate equal to $\mu$. Regarding the cost, roughly speaking, the idea is to penalize high levels of demand and the controller whenever it slows down the demand by choosing a lower intensity. It is also supposed that an immediate (boundary) cost is incurred whenever a project is finished, and that there are no costs when the system is in the standby situation.

As the state space of the process we take $E=\bigcup_{i \in \mathbb{N}}[\{i\} \times[0, \tau)]$. A point $(0, t) \in E$ denotes that the system is in standby. A point $(i, t) \in E$ with $i \geq 1$ indicates that the level of demand for the system is $i$, and the amount of elapsed time of the present project is $t$. For all $x \in E, \mathbb{U}(x)=\mathbb{U}=\left[\lambda_{a}, \lambda_{b}\right]$. The costs are defined for $x=(i, t) \in E$ and $a \in \mathbb{U}$ by $f(x, a)=\alpha_{i}+h_{i}(a)$ and $r(x, a)=r_{i}$, where $\left(\alpha_{i}\right)_{i \in \mathbb{N}}$ is an increasing sequence of nonnegative real numbers satisfying, for each $i \in \mathbb{N}, \alpha_{i} \leq i \alpha$ for a constant $\alpha, h_{i}(a)$ is a decreasing mapping in $a$ with nonnegative values satisfying $h_{i}(a) \leq i \psi$ for a constant $\psi$, and $\left(r_{i}\right)_{i \in \mathbb{N}}$ is a sequence of nonnegative real numbers satisfying $r_{i} \leq i \xi$ for a constant $\xi$. The flow $\phi$ of the PDMP is defined by $\phi(x, s)=(i, t+s)$ for $x=(i, t) \in E$ and $s \leq \tau-t$. For $t \in[0, \tau)$ and $a \in \mathbb{U}$, the intensity of the jump is given by $\lambda((i, t), a)=a$ when $i \geq 1$ and by $\lambda(x, a)=\mu$ when $i=0$. Finally, the transition measure is defined by $Q((0, t), a ; A)=\delta_{\{(1,0)\}}(A)$ and $Q((i, t), a ; A)=\delta_{\{(i+1, t)\}}(A)$ when $t \in[0, \tau)$, and by $Q((0, \tau), a ; A)=\delta_{\{(0,0)\}}(A)$ and $Q((i, \tau), a ; A)=\delta_{\{(i-1,0)\}}(A)$. The standby situation of the system is represented by the set $\{0\} \times[0, \tau)$ for mathematical convenience. It should be noted that it does not affect the optimization problem due to the memoryless property of the exponential distribution. Finally, we assume the classical stability condition: $\lambda_{b} \tau<1$ (see [5, Proposition 34.36]).

We now show that all the assumptions of the paper are satisfied for this example. Assumptions 2.1, 2.2, and 2.4 are trivially satisfied. There exists a set $A=\left\{y \in E: t_{*}(y)=\tau\right\}$ such that, for all $z \in \partial E$ and all $a \in \mathbb{U}(z), Q(z, a ; A)=1$. Moreover, for all $x \in E$ and $a \in \mathbb{U}(x)$, $\lambda(x, a) \leq \lambda_{b}$. Consequently, the hypotheses of Proposition 24.6 of [5] are satisfied, implying that Assumption 2.3 is satisfied.

Since $\mathbb{U}(x)$ does not depend on $x$, Assumption 2.5 is clearly satisfied. Now, observe that, for all $x \in E, t_{*}(x) \leq \tau<\infty$. For all $x \in E$, the mappings $\lambda(x, \cdot)$ are continuous on $\mathbb{U}(x)=\left[\lambda_{a}, \lambda_{b}\right]$. Taking, for $x \in E, \bar{\lambda}(x)=\lambda_{b}$ we have

$$
\int_{0}^{t} \bar{\lambda}(\phi(x, s)) \mathrm{d} s \leq \int_{0}^{t_{*}(x)} \bar{\lambda}(\phi(x, s)) \mathrm{d} s=\lambda_{b} \tau<\infty
$$

showing that Assumption 3.3 is satisfied.

By definition, $f(x, \cdot)$ and $r(x, \cdot)$ are continuous on $\mathbb{U}(x)=\left[\lambda_{a}, \lambda_{b}\right]$ for all $x \in E$, implying that Assumption 3.4 is satisfied. Here $Q$ does not depend on the control and, consequently, Assumption 3.5 is trivially satisfied. Define $\underline{\lambda}(x)=\lambda_{a}, \bar{f}(x)=(\alpha+\psi) i$ for $x=(i, t) \in E$, and $K_{\lambda}=\tau \mathrm{e}^{c \tau}$. Then we have $\lambda(x, a) \geq \underline{\lambda}(x), f(x, a) \leq \bar{f}(x)$, 


$$
\begin{aligned}
\int_{0}^{t_{*}(x)} \exp \{c t- & \left.\int_{0}^{t} \underline{\lambda}(\phi(x, s)) \mathrm{d} s\right\} \mathrm{d} t \leq \tau \mathrm{e}^{c \tau}=K_{\lambda}, \text { and } \\
& \int_{0}^{t_{*}(x)} \exp \left\{-\int_{0}^{t} \underline{\lambda}(\phi(x, s)) \mathrm{d} s\right\} \bar{f}(\phi(x, t)) \mathrm{d} t \leq \bar{f}(x) \tau<\infty .
\end{aligned}
$$

Recalling that $t_{*}(x) \leq \tau<\infty$ for all $x \in E$, this shows that Assumption 3.6(a)-(e) are satisfied.

It remains to show that Assumptions 3.1 and 3.2 are satisfied. In order to verify these assumptions, we need to appropriately define the test functions $g$ and $\bar{r}$. We first need the following proposition.

Proposition 5.1. There exist $d_{1}>0, d_{2}>0$, and $c>0$ such that

$$
\begin{gathered}
\mathrm{e}^{d_{1}} \frac{\lambda_{b}}{\lambda_{b}+d_{2}}<\mathrm{e}^{-d_{1}+d_{2} \tau}<1, \\
c<d_{2}-\lambda_{b}\left(\mathrm{e}^{d_{1}}-1\right), \\
\mathrm{e}^{d_{1}}\left(1-\mathrm{e}^{-\mu \tau}\right)-\mathrm{e}^{-a_{1}} \leq 0 .
\end{gathered}
$$

Proof. Consider the function $c(z)=z / \tau-\lambda_{b}\left(\mathrm{e}^{z}-1\right)$. Then, under the assumption that $\lambda_{b} \tau<1$, we can find a $z_{0}>0$ such that $c\left(z_{0}\right)>0$ and $1-\mathrm{e}^{-\mu \tau} \leq \mathrm{e}^{-2 z_{0}}$ by observing that $c(0)=0$ and $c^{\prime}(0)>0$. This implies that we can find a $0<\varepsilon<z_{0} / 2$ such that $c\left(z_{0}\right)-2 \varepsilon / \tau=\left(z_{0}-2 \varepsilon\right) / \tau-\lambda_{b}\left(\mathrm{e}^{z_{0}}-1\right)>0$. We set $d_{1}=z_{0}-\varepsilon>0$ and $d_{2}=\left(z_{0}-2 \varepsilon\right) / \tau>0$. Since $1-\mathrm{e}^{-\mu \tau} \leq \mathrm{e}^{-2 z_{0}}$, we have $1-\mathrm{e}^{-\mu \tau} \leq \mathrm{e}^{-2 z_{0}+2 \varepsilon}=\mathrm{e}^{-2 a_{1}}$, giving (5.2). Note that $-d_{1}+d_{2} \tau=-\left(z_{0}-\varepsilon\right)+z_{0}-2 \varepsilon=-\varepsilon$ and, thus, $\mathrm{e}^{-d_{1}+d_{2} \tau}=\mathrm{e}^{-\varepsilon}<1$. From $c\left(z_{0}\right)-2 \varepsilon / \tau>0$ we obtain $d_{2}+\lambda_{b}>\lambda_{b} \mathrm{e}^{d_{1}+\varepsilon}$ and, thus, $\mathrm{e}^{d_{1}} \lambda_{b} /\left(\lambda_{b}+d_{2}\right)<\mathrm{e}^{-d_{1}+d_{2} \tau}$, giving (5.1). Moreover, choosing $c>0$ such that $c<c\left(z_{0}\right)-2 \varepsilon / \tau$ we obtain $c<d_{2}-\lambda_{b}\left(\mathrm{e}^{d_{1}+\varepsilon}-1\right)<d_{2}-\lambda_{b}\left(\mathrm{e}^{d_{1}}-1\right)$, yielding (5.3).

We can now define the test functions $g$ and $\bar{r}$, and the parameters required in Assumptions 3.1 and 3.2. Consider $d_{1}, d_{2}$, and $c$ satisfying (5.1) and (5.2), and define $b=\mathrm{e}^{d_{2} \tau}\left(\mu\left[\mathrm{e}^{d_{1}}-1\right]+c\right)>$ $0, \delta=c, d_{3}=1-\mathrm{e}^{-d_{1}+d_{2} \tau}>0, M=\max \left((\alpha+\psi) / d_{1}, 2 c \xi / d_{3} \mathrm{~d}_{1}\right)$, and

$$
\begin{aligned}
& g(x)= \begin{cases}\mathrm{e}^{d_{2} \tau} & \text { if } x=(0, t) \in E, \\
\mathrm{e}^{d_{1} i+d_{2}(\tau-t)} & \text { if } x=(i, t) \in E \text { and } i \geq 1,\end{cases} \\
& \bar{r}(z)= \begin{cases}0 & \text { if } z=(0, \tau), \\
d_{3} \mathrm{e}^{d_{1} i} & \text { if } z=(i, \tau) \text { and } i \geq 1 .\end{cases}
\end{aligned}
$$

For the constants $b, c, \delta$, and $M$ defined previously, we have the following result.

Proposition 5.2. The functions $g$ and $\bar{r}$ satisfy Assumption 3.1.

Proof. First consider $x=(0, t) \in E$ and $z=(0, \tau)$. Then in this case $\mathcal{X} g(x)=0$ and

$$
\chi g(x)+c g(x)-\lambda(x, a)[g(x)-Q g(x, a)]=\mathrm{e}^{d_{2} \tau}\left(\mu\left[\mathrm{e}^{d_{1}}-1\right]+c\right)=b,
$$

and so (3.1) holds. Clearly, $f(x, a)=0 \leq M g(x)$ and so (3.2) is satisfied. Since $\bar{r}(z)=0$, we have $\bar{r}(z)+Q g(z, a)=\mathrm{e}^{d_{2} \tau}=g(z)$, showing (3.3). Finally, (3.4) is trivially satisfied since $r(z, a)=0$. Now consider $x=(i, t) \in E$ and $z=(i, \tau)$ with $i \geq 1$. Note that $\chi g(x)=-d_{2} g(x)$ and

$$
X g(x)+c g(x)-\lambda(x, a)[g(x)-Q g(x, a)]=\mathrm{e}^{d_{1} i+d_{2}(\tau-t)}\left(c-d_{2}+a\left(\mathrm{e}^{d_{1}}-1\right)\right) .
$$


From (5.2), it follows that

$$
\max _{a \in\left[\lambda_{a}, \lambda_{b}\right]}\left\{\mathrm{e}^{d_{1} i+d_{2}(\tau-t)}\left(c-d_{2}+a\left(\mathrm{e}^{d_{1}}-1\right)\right)\right\} \leq \mathrm{e}^{d_{1} i+d_{2}(\tau-t)}\left(c-d_{2}+\lambda_{b}\left(\mathrm{e}^{d_{1}}-1\right)\right) \leq 0,
$$

yielding (3.1). Moreover, $f(x, a) \leq(\alpha+\psi) i \leq M g(x)$, showing (3.2). Equation (3.3) also holds since $\bar{r}(z)+Q g(z, a)=d_{3} \mathrm{e}^{\bar{d}_{1} i}+\mathrm{e}^{d_{1}(i-1)+d_{2} \tau}=\mathrm{e}^{d_{1} i} \leq g(z)$. Finally, $r(z, a)=r_{i} \leq$ $\xi i \leq M \bar{r}(z)$, implying (3.4) and completing the proof.

Next we want to show that Assumption 3.2 is satisfied. We first need the following proposition.

Proposition 5.3. Set $\beta=\mathrm{e}^{-d_{1}+d_{2} \tau}<1$ (see (5.1)). For any $u \in 8_{\mathbb{U}}$, we have

$$
\begin{gathered}
G g\left(x, u_{\phi}\right) \leq \beta g(x) \quad \text { for } x=(i, t) \in E, \text { with } i \geq 1, \\
G g\left(x, u_{\phi}\right) \leq \beta g(x)+l(x) g(0,0) \quad \text { for } x=(0, t) \in E,
\end{gathered}
$$

where $l(x)=\mathrm{e}^{-\mu(\tau-t)}$ for $x=(0, t)$ and $l(x)=0$ otherwise.

Proof. Let us first show that (5.4) holds. Consider $u \in \delta_{\mathbb{U}}$ and $x=(i, t) \in E$, with $i \geq 1$ fixed. For notational simplicity, we write $\lambda_{i}(t+s)=\lambda(\phi(x, s), u(\phi(x, s)))$ and $\Lambda_{i}(t, s)=\int_{0}^{s} \lambda(\phi(x, v), u(\phi(x, v))) \mathrm{d} v$. Noting that $\lambda_{i}(t+s) / \lambda_{b} \leq 1$ we have, from (5.1),

$$
\begin{aligned}
G g\left(x, u_{\phi}\right)= & \mathrm{e}^{d_{1} i}\left\{\mathrm{e}^{d_{1}+d_{2}(\tau-t)} \int_{0}^{\tau-t} \lambda_{i}(t+s) \mathrm{e}^{-\left(\Lambda_{i}(t, s)+d_{2} s\right)} \mathrm{d} s+\mathrm{e}^{-\Lambda_{i}(t, \tau-t)} \mathrm{e}^{-d_{1}+d_{2} \tau}\right\} \\
= & g(i, t)\left\{\mathrm{e}^{d_{1}} \frac{\lambda_{b}}{\lambda_{b}+d_{2}} \int_{0}^{\tau-t}\left(1+\frac{d_{2}}{\lambda_{b}}\right) \lambda_{i}(t+s) \mathrm{e}^{-\left(\Lambda_{i}(t, s)+d_{2} s\right)} \mathrm{d} s\right. \\
& \left.\quad+\mathrm{e}^{-\Lambda_{i}(t, \tau-t)-d_{2}(\tau-t)} \mathrm{e}^{-d_{1}+d_{2} \tau}\right\} \\
\leq & \beta g(i, t)\left\{\int_{0}^{\tau-t}\left(\lambda_{i}(t+s)+d_{2}\right) \mathrm{e}^{-\left(\Lambda_{i}(t, s)+d_{2} s\right)} \mathrm{d} s+\mathrm{e}^{-\Lambda_{i}(t, \tau-t)-d_{2}(\tau-t)}\right\} \\
= & \beta g(i, t),
\end{aligned}
$$

showing (5.4).

Now, for $x=(0, t) \in E$, we have

$$
\begin{aligned}
G g\left(x, u_{\phi}\right) & =\mathrm{e}^{d_{1}+d_{2} \tau}\left[1-\mathrm{e}^{-\mu(\tau-t)}\right]+\mathrm{e}^{d_{2} \tau} \mathrm{e}^{-\mu(\tau-t)} \\
& =\beta g(x)-\mathrm{e}^{-d_{1}+d_{2} \tau} \mathrm{e}^{d_{2} \tau}+\mathrm{e}^{d_{1}+d_{2} \tau}\left[1-\mathrm{e}^{-\mu(\tau-t)}\right]+l(x) g(0,0),
\end{aligned}
$$

since $\mathrm{e}^{d_{2} \tau} \mathrm{e}^{-\mu(\tau-t)}=l(x) v(g)$. From (5.3) we obtain

$$
\mathrm{e}^{d_{1}+d_{2} \tau}\left[1-\mathrm{e}^{-\mu(\tau-t)}\right]-\mathrm{e}^{-d_{1}+d_{2} \tau} \mathrm{e}^{d_{2} \tau} \leq 0,
$$

showing (5.5) and completing the proof.

The next proposition shows that Assumption 3.2 is satisfied.

Proposition 5.4. For any $u \in \S_{\mathbb{U}}$, there exists a probability measure $v_{u}$ such that Assumption 3.2 is satisfied.

Proof. Clearly, it is easy to see that, for a fixed $u \in \mathcal{S}_{\mathbb{U}}$, the Markov kernel $G\left(\cdot, u_{\phi} ; \cdot\right)$ is irreducible. Moreover, we have $G\left(x, u_{\phi} ; A\right) \geq l(x) \delta_{(0,0)}(A)$, where the function $l$ has been defined in Proposition 5.3. Define the set $C=\{(0, t): 0 \leq t<\tau\}$. Therefore, we have, for all $x \in C, G\left(x, u_{\phi} ; A\right) \geq \mathrm{e}^{-\mu \tau} \delta_{(0,0)}(A)$, implying that the set $C$ is a petite set; see [14, p. 121]. 
Now since $\beta<1$, we find from Proposition 5.3 that there exists a constant $K$ such that

$$
G g\left(x, u_{\phi}\right) \leq g(x)+K \mathbf{1}_{C}(x) .
$$

Combining Theorem 4.1(i) of [13] and Theorem 11.0.1 of [14], the previous inequality shows that the Markov kernel $G g\left(\cdot, u_{\phi} ; \cdot\right)$ is positive Harris recurrent. Consequently, there exists a unique invariant probability measure for $G g\left(\cdot, u_{\phi} ; \cdot\right)$. Note that $g(x) \geq 1$, and $0 \leq l(x) \leq 1$. Moreover, for any $u \in \delta_{\mathbb{U}}$ and $x \in E, G\left(x, u_{\phi}, A\right) \geq l(x) \delta_{(0,0)}(A), \int_{E} l(y) \delta_{(0,0)}(\mathrm{d} y)>0$, and $\int_{E} g(y) \delta_{(0,0)}(\mathrm{d} y)=g((0,0))<\infty$. Now, from Proposition 5.3, it follows that, for any $u \in \delta_{\mathbb{U}}$ and $x \in E, G g\left(x, u_{\phi}\right) \leq \beta g(x)+l(x) \int_{E} g(y) \delta_{(0,0)}(\mathrm{d} y)$, implying that the hypotheses and items (i)-(iv) of Proposition 10.2.5 of [11] are satisfied. Consequently, by applying Proposition 10.2.5 of [11], the result follows.

\section{Appendix A}

In this appendix we present the proofs of the main results of the paper.

\section{A.1. Proof of Theorem 4.1}

The next two propositions establish a connection between a general integro-differential inequality (respectively equality) related to the local characteristics of the PDMP and an inequality (respectively equality) related to the operators $G_{\alpha}, L_{\alpha}$, and $H_{\alpha}$. They will be crucial for the boundedness results on $\mathscr{g}_{D}^{\alpha}(\cdot)$ to be developed in the sequel.

Proposition A.1. Suppose that there exist $v \in \mathbb{M}^{a c}\left(E, \mathbb{R}_{+}\right), \ell \in \mathbb{M}(E)^{+}, k \in \mathbb{M}(E)^{+}, p \in$ $\mathbb{M}(\partial E)^{+}, \Theta=\left(\mu, \mu_{\partial}\right) \in \delta_{\mathbb{V}^{r}}, d \geq 0$, and $\alpha \geq-c$ satisfying

$$
\begin{aligned}
X v(\phi(x, t))-[\alpha+\lambda(\phi(x, t), \mu(x, t))] v(\phi(x, t))+\ell(\phi(x, t)) & \\
& +\lambda(\phi(x, t), \mu(x, t)) Q k(\phi(x, t), \mu(x, t)) \leq d
\end{aligned}
$$

for all $x \in E, t \in\left[0, t_{*}(x)\right)$, and

$$
v\left(\phi\left(x, t_{*}(x)\right)\right) \geq p\left(\phi\left(x, t_{*}(x)\right)\right)+Q k\left(\phi\left(x, t_{*}(x)\right), \mu_{\partial}\left(\phi\left(x, t_{*}(x)\right)\right)\right)
$$

for all $x \in E$ with $t_{*}(x)<\infty$. Then

$$
v(x) \geq-d \mathcal{L}_{\alpha}(x, \Theta(x))+L_{\alpha} \ell(x, \Theta(x))+H_{\alpha} p(x, \Theta(x))+G_{\alpha} k(x, \Theta(x)) .
$$

Proof. Multiplying both sides of (A.1) by $\mathrm{e}^{-\alpha t-\Lambda^{\mu(x)}(x, t)}$ and integrating over $[0, s]$ for $s \in\left[0, t_{*}(x)\right)$ we obtain

$$
\begin{aligned}
& d \int_{0}^{s} \mathrm{e}^{-\alpha t-\Lambda^{\mu(x)}(x, t)} \mathrm{d} t \\
& \quad \geq \mathrm{e}^{-\alpha s-\Lambda^{\mu(x)}(x, s)} v(\phi(x, s))-v(x) \\
& \quad+\int_{0}^{s} \mathrm{e}^{-\alpha t-\Lambda^{\mu(x)}(x, t)}[\ell(\phi(x, t))+\lambda(\phi(x, t), \mu(x, t)) Q k(\phi(x, t), \mu(x, t))] \mathrm{d} t .
\end{aligned}
$$

Consider the case in which $t_{*}(x)<\infty$. By using the fact that $v \in \mathbb{M}^{a c}(E)$ we obtain from Remark 3.1(ii) and (A.4) that

$$
\begin{aligned}
v(x) \geq & -d \mathcal{L}_{\alpha}(x, \Theta(x))+L_{\alpha} \ell(x, \Theta(x))+\mathrm{e}^{-\alpha t_{*}(x)-\Lambda^{\mu(x)}\left(x, t_{*}(x)\right)} v\left(\phi\left(x, t_{*}(x)\right)\right) \\
& +\int_{0}^{t_{*}(x)} \mathrm{e}^{-\alpha t-\Lambda^{\mu(x)}(x, t)} \lambda(\phi(x, t), \mu(x, t)) Q k(\phi(x, t), \mu(x, t)) \mathrm{d} t
\end{aligned}
$$


However, from (A.2), it follows that

$$
v(x) \geq-d \mathcal{L}_{\alpha}(x, \Theta(x))+L_{\alpha} \ell(x, \Theta(x))+H_{\alpha} p(x, \Theta(x))+G_{\alpha} k(x, \Theta(x)) .
$$

Now consider the case in which $t_{*}(x)=+\infty$. From (A.4) (and recalling that $v$ is positive) we have

$$
\begin{aligned}
& d \int_{0}^{s} \mathrm{e}^{-\alpha t-\Lambda^{\mu(x)}(x, t)} \mathrm{d} t \\
& \quad \geq-v(x)+\int_{0}^{s} \mathrm{e}^{-\alpha t-\Lambda^{\mu(x)}(x, t)}[\ell(\phi(x, t))+\lambda(\phi(x, t), \mu(x, t)) Q k(\phi(x, t), \mu(x, t))] \mathrm{d} t,
\end{aligned}
$$

and so, by taking the limit as $s$ tends to $\infty$ in the previous equation, we obtain

$$
\begin{aligned}
v(x) \geq & -d \mathcal{L}_{\alpha}(x, \Theta(x))+L_{\alpha} \ell(x, \Theta(x)) \\
& +\int_{0}^{t_{*}(x)} \mathrm{e}^{-\alpha t-\Lambda^{\mu(x)}(x, t)} \lambda(\phi(x, t), \mu(x, t)) Q k(\phi(x, t), \mu(x, t)) \mathrm{d} t .
\end{aligned}
$$

However, by using the fact that $t_{*}(x)=+\infty$ and Remark 3.1(i), we have $H_{\alpha} p(x, \Theta(x))=0$ and

$$
G_{\alpha} k(x, \Theta(x))=\int_{0}^{t_{*}(x)} \mathrm{e}^{-\alpha t-\Lambda^{\mu(x)}(x, t)} \lambda(\phi(x, t), \mu(x, t)) Q k(\phi(x, t), \mu(x, t)) \mathrm{d} t .
$$

This completes the proof.

If the inequalities in (A.1) and (A.2) are replaced by equalities then the hypotheses of Proposition A.1 must be restricted to $\alpha \geq 0$ to show that the inequality in (A.3) becomes an equality; more specifically, we have the following result.

Proposition A.2. Suppose that there exist $v \in \mathbb{M}_{w}^{a c}\left(E, \mathbb{R}_{+}\right), \ell \in \mathbb{M}(E)^{+}, k \in \mathbb{M}(E)^{+}, p \in$ $\mathbb{M}(\partial E)^{+}, \Theta=\left(\mu, \mu_{\partial}\right) \in \delta_{\mathbb{V}^{r}}, d \geq 0$, and $\alpha \geq 0$ satisfying

$$
\begin{aligned}
X v(\phi(x, t))-[\alpha+\lambda(\phi(x, t), \mu(x, t))] v & (\phi(x, t))+\ell(\phi(x, t)) \\
& +\lambda(\phi(x, t), \mu(x, t)) Q k(\phi(x, t), \mu(x, t))=d
\end{aligned}
$$

for all $x \in E, t \in\left[0, t_{*}(x)\right)$, and

$$
v\left(\phi\left(x, t_{*}(x)\right)\right)=p\left(\phi\left(x, t_{*}(x)\right)\right)+Q k\left(\phi\left(x, t_{*}(x)\right), \mu_{\partial}\left(\phi\left(x, t_{*}(x)\right)\right)\right)
$$

for all $x \in E$ with $t_{*}(x)<\infty$. Then

$$
v(x)=-d \mathcal{L}_{\alpha}(x, \Theta(x))+L_{\alpha} \ell(x, \Theta(x))+H_{\alpha} p(x, \Theta(x))+G_{\alpha} k(x, \Theta(x)) .
$$

Proof. By following the same steps as in the first part of the proof of Proposition A.1 we have, for all $s \in\left[0, t_{*}(x)\right)$,

$$
\begin{aligned}
d \int_{0}^{s} \mathrm{e}^{-\alpha t-\Lambda^{\mu(x)}(x, t)} \mathrm{d} t \\
=\mathrm{e}^{-\alpha s-\Lambda^{\mu(x)}(x, s)} v(\phi(x, s))-v(x) \\
\quad+\int_{0}^{s} \mathrm{e}^{-\alpha t-\Lambda^{\mu(x)}(x, t)}[\ell(\phi(x, t))+\lambda(\phi(x, t), \mu(x, t)) Q k(\phi(x, t), \mu(x, t))] \mathrm{d} t .
\end{aligned}
$$


The case in which $t_{*}(x)<\infty$ can be treated in the same manner as in the proof of Proposition A.1. However, the case in which $t_{*}(x)=+\infty$ is different. By using Assumption 3.6(d) and the fact that $0 \leq v \leq\|v\|_{g} g$, we have, for any $\alpha \geq 0$,

$$
\lim _{s \rightarrow+\infty} \mathrm{e}^{-\alpha s-\Lambda^{\mu(x)}(x, s)} v(\phi(x, s)) \leq\|v\|_{g} \lim _{s \rightarrow+\infty} \exp \left\{-\int_{0}^{t_{*}(x)} \underline{\lambda}(\phi(x, t)) \mathrm{d} t\right\} g(\phi(x, s))=0 .
$$

Therefore, taking the limit as $s$ tends to $\infty$ in (A.6), we have

$$
\begin{aligned}
d \mathscr{L}_{\alpha}(x, \Theta(x))= & -v(x)+L_{\alpha} \ell(x, \Theta(x)) \\
& +\int_{0}^{s} \mathrm{e}^{-\alpha t-\Lambda^{\mu(x)}(x, t)} \lambda(\phi(x, t), \mu(x, t)) Q k(\phi(x, t), \mu(x, t)) \mathrm{d} t,
\end{aligned}
$$

and, using Remark 3.1(i), this proves (A.5).

Applying Proposition A.1 to inequalities (3.1) and (3.3) we obtain the following corollary.

Corollary A.1. For any $u \in \S_{\mathbb{U}}, \alpha \geq-c$, and $x \in E$,

$$
g(x) \geq-b \mathcal{L}_{\alpha}\left(x, u_{\phi}(x)\right)+(c+\alpha) L_{\alpha} g\left(x, u_{\phi}(x)\right)+H_{\alpha} \bar{r}\left(x, u_{\phi}(x)\right)+G_{\alpha} g\left(x, u_{\phi}(x)\right),
$$

and, for all $\Theta \in \delta_{\mathbb{V} r}$,

$$
(c+\alpha) L_{\alpha} g(x, \Theta(x))+H_{\alpha} \bar{r}(x, \Theta(x))+G_{\alpha} g(x, \Theta(x)) \leq b K_{\lambda}+g(x) .
$$

Proof. Clearly, from Proposition 3.8 and Remark 3.11 of [2], it follows that $u_{\phi} \in \delta_{\mathbb{V} r}$. Consequently, setting $d=b, v=g, \ell=(c+\alpha) g, p=\bar{r}, k=g$, and $\Theta=u_{\phi}$ in Proposition A.1, we obtain (A.7). Similarly, from Remark 3.1(ii), inequality (A.8) is a straightforward consequence of inequality (A.3).

The next theorem provides bounds in terms of $\alpha$ and $g$ for a sequence of functions defined by a general recursive equation and for the functions $L f, H r$, and $L g$.

Theorem A.1. Define the sequence $\left(q_{m}(x)\right)_{m \in \mathbb{N}}$ by

$$
\begin{gathered}
q_{0}^{\alpha}(x)=0, \\
q_{m+1}^{\alpha}(x)=L_{\alpha} f\left(x, u_{\phi}^{m+1}(x)\right)+H_{\alpha} r\left(x, u_{\phi}^{m+1}(x)\right)+G_{\alpha} q_{m}^{\alpha}\left(x, u_{\phi}^{m+1}(x)\right),
\end{gathered}
$$

where $x \in E,\left(u^{m}\right)_{m \in \mathbb{N}} \in \delta_{\mathbb{U}}$, and $\alpha>0$. Then the following assertions hold:

(i) for any $x \in E, m \in \mathbb{N}$, and $\alpha \in[0, \delta)$, we have

$$
q_{m}^{\alpha}(x) \leq \frac{M}{c+\alpha} g(x)+\frac{M b}{c \alpha},
$$

(ii) for any $x \in E$ and $u \in \mathcal{S}_{\mathbb{U}}$,

$$
\begin{gathered}
0 \leq L f\left(x, u_{\phi}(x)\right)+H r\left(x, u_{\phi}(x)\right) \leq \frac{M\left(1+b K_{\lambda}\right)}{c} g(x), \\
0 \leq \operatorname{Lg}\left(x, u_{\phi}(x)\right) \leq \frac{\left(1+b K_{\lambda}\right)}{c} g(x) .
\end{gathered}
$$


Proof. Let us show (A.10) by induction. For $m=0$, it is immediate since $q_{0}^{\alpha}=0$. Suppose that it holds for $m$. Combining (A.9) and (A.10) we have

$$
\begin{aligned}
q_{m+1}^{\alpha}(x) \leq & L_{\alpha} f\left(x, u_{\phi}^{m}(x)\right)+H_{\alpha} r\left(x, u_{\phi}^{m}(x)\right)+\frac{M}{c+\alpha} G_{\alpha} g\left(x, u_{\phi}^{m}(x)\right) \\
& +\frac{M b}{c \alpha} G_{\alpha} 1\left(x, u_{\phi}^{m}(x)\right) .
\end{aligned}
$$

Moreover, from (A.7) and (A.8), we obtain

$$
G_{\alpha} g\left(x, u_{\phi}^{m}(x)\right) \leq g(x)+b \mathscr{L}_{\alpha}\left(x, u_{\phi}^{m}(x)\right)-(c+\alpha) L_{\alpha} g\left(x, u_{\phi}^{m}(x)\right)-H_{\alpha} \bar{r}\left(x, u_{\phi}^{m}(x)\right) .
$$

Substituting (A.14) into (A.13) and using (3.2) and (3.4), we obtain

$$
\begin{aligned}
q_{m+1}^{\alpha}(x) \leq & L_{\alpha}(f-M g)\left(x, u_{\phi}^{m}(x)\right)+H_{\alpha}\left(r-\frac{M}{c+\alpha} \bar{r}\right)\left(x, u_{\phi}^{m}(x)\right)+\frac{M}{c+\alpha} g(x) \\
& +M b\left(\frac{1}{c \alpha} G_{\alpha} 1\left(x, u_{\phi}^{m}(x)\right)+\frac{1}{c+\alpha} \mathcal{L}_{\alpha}\left(x, u_{\phi}^{m}(x)\right)\right) \\
\leq & \frac{M}{c+\alpha} g(x)+\frac{M b}{c \alpha}\left(G_{\alpha} 1\left(x, u_{\phi}^{m}(x)\right)+\alpha \mathcal{L}_{\alpha}\left(x, u_{\phi}^{m}(x)\right)\right) \\
\leq & \frac{M}{c+\alpha} g(x)+\frac{M b}{c \alpha}
\end{aligned}
$$

since $G_{\alpha} 1\left(x, u_{\phi}^{m}(x)\right)+\alpha \mathcal{L}_{\alpha}\left(x, u_{\phi}^{m}(x)\right)=1$.

Let us now show (A.11) and (A.12). For $\alpha=0$, it follows from Remark 3.1(ii) and (A.7) that

$$
\begin{aligned}
g(x)+b K_{\lambda} & \geq g(x)+b \mathcal{L}\left(x, u_{\phi}(x)\right) \\
& \geq c L g\left(x, u_{\phi}(x)\right)+H \bar{r}\left(x, u_{\phi}(x)\right)+G g\left(x, u_{\phi}(x)\right),
\end{aligned}
$$

showing (A.12) since $g \geq 1$ and $\bar{r} \geq 0$. Now, combining (3.2), (3.4), and (A.15) we obtain (A.11), showing the last part of the result.

Proof of Theorem 4.1. By using Propositions 7.1 and 7.5 of [2], it can be shown that there exists $u_{\phi}^{m} \in \delta_{\mathbb{V}}$ such that the sequence $\left(v_{m}^{\alpha}(x)\right)_{m \in \mathbb{N}}$ defined by $v_{m+1}^{\alpha}(x)=L_{\alpha} f\left(x, u_{\phi}^{m}(x)\right)+$ $H_{\alpha} r\left(x, u_{\phi}^{m}(x)\right)+G_{\alpha} v_{m}^{\alpha}\left(x, u_{\phi}^{m}(x)\right)$ and $v_{0}^{\alpha}(x)=0$ satisfies $v_{m+1}^{\alpha} \uparrow \mathcal{g}_{D}^{\alpha}(x)$ as $m \uparrow \infty$. Therefore, considering $q_{m}^{\alpha}=v_{m}^{\alpha}$ in Theorem A.1 and taking the limit as $m \uparrow \infty$, we obtain (4.1).

\section{A.2. Proof of Theorem 4.2}

The following technical lemma shows that $g_{D}^{\alpha}(x)$ can be written as an infinite sum of iterates of the stochastic kernel $G_{\alpha}$. Using this result, $g_{D}^{\alpha}(x)$ is characterized in terms of the Markov kernel $G$ in Proposition A.3. This is an important property. Indeed, by using classical hypotheses on $G$ such as the geometric ergodic condition in Assumption 3.2, it will be shown in Theorem 4.2 that the mapping defined by $g_{D}^{\alpha}(\cdot)-g_{D}^{\alpha}(y)$ for $y$ fixed in $E$ belongs to $\mathbb{B}_{g}(E)$.

Lemma A.1. For each $\alpha>0$, there exists $u^{\alpha} \in \S_{\mathbb{U}}$ such that

$$
\mathcal{g}_{D}^{\alpha}(x)=\sum_{k=0}^{\infty} G_{\alpha}^{k}\left(L_{\alpha} f+H_{\alpha} r\right)\left(x, u_{\phi}^{\alpha}(x)\right) .
$$


Proof. As shown in [2, Theorem 7.5], $\mathscr{\mathcal { F }}_{\mathscr{D}}^{\alpha} \in \mathbb{M}(\mathbb{E})$ and $\mathscr{g}_{\mathscr{D}}^{\alpha}(x)=\mathcal{R}_{\alpha}\left(0, \mathcal{G}_{\mathscr{D}}^{\alpha}\right)(x)$. Moreover, from Theorem 6.4 of [2], there exists $u^{\alpha} \in \mathcal{S}_{\mathbb{U}}$ such that the ordinary feedback measurable selector $u_{\phi}^{\alpha} \in \delta_{\mathbb{V}}$ satisfies

$$
\begin{aligned}
\mathscr{g}_{D}^{\alpha}(x) & =\mathcal{R}_{\alpha}\left(0, \mathscr{g}_{D}^{\alpha}\right)(x) \\
& =\mathcal{T}_{\alpha}\left(0, \mathscr{g}_{D}^{\alpha}\right)(x) \\
& =L_{\alpha} f\left(x, u_{\phi}^{\alpha}\right)(x)+H_{\alpha} r\left(x, u_{\phi}^{\alpha}\right)+G_{\alpha} \mathscr{g}_{D}^{\alpha}\left(x, u_{\phi}^{\alpha}\right) .
\end{aligned}
$$

Iterating (A.16) and recalling that $\mathcal{G}_{D}^{\alpha}(y) \geq 0$ for every $y$ yields, for every $m \in \mathbb{N}$,

$$
\begin{aligned}
\mathscr{g}_{D}^{\alpha}(x) & =\sum_{k=0}^{m-1} G_{\alpha}^{k}\left(L_{\alpha} f+H_{\alpha} r\right)\left(x, u_{\phi}^{\alpha}(x)\right)+G_{\alpha}^{m} \mathcal{g}_{\mathcal{D}}^{\alpha}\left(x, u_{\phi}^{\alpha}(x)\right) \\
& \geq \sum_{k=0}^{m-1} G_{\alpha}^{k}\left(L_{\alpha} f+H_{\alpha} r\right)\left(x, u_{\phi}^{\alpha}(x)\right) .
\end{aligned}
$$

For the control $U_{u_{\phi}^{\alpha}} \in \mathcal{U}$ (see Definition 2.3), it is easy to show that

$$
\begin{aligned}
\sum_{k=0}^{m-1} G_{\alpha}^{k}\left(L_{\alpha} f\right. & \left.+H_{\alpha} r\right)\left(x, u_{\phi}^{\alpha}(x)\right) \\
=\mathrm{E}_{(x, 0)}^{U_{u_{\phi}^{\alpha}}}[ & {\left[\int_{0}^{T_{m}} \mathrm{e}^{-\alpha s} f(X(s), u(N(s), Z(s), \tau(s))) \mathrm{d} s\right.} \\
& \left.+\int_{0}^{T_{m}} \mathrm{e}^{-\alpha s} r\left(X(s-), u_{\partial}(N(s-), Z(s-))\right) \mathrm{d} p^{*}(s)\right],
\end{aligned}
$$

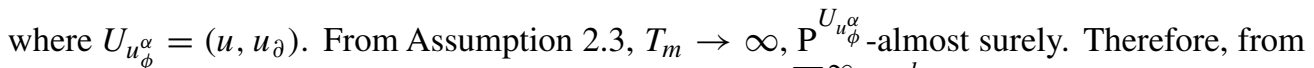
the monotone convergence theorem, (A.18) implies that $\sum_{k=0}^{\infty} G_{\alpha}^{k}\left(L_{\alpha} f+H_{\alpha} r\right)\left(x, u_{\phi}^{\alpha}(x)\right)=$ $D^{\alpha}\left(U_{u_{\phi}^{\alpha}}, x\right)$, and from (A.17),

$$
\mathscr{g}_{D}^{\alpha}(x) \geq \sum_{k=0}^{\infty} G_{\alpha}^{k}\left(L_{\alpha} f+H_{\alpha} r\right)\left(x, u_{\phi}^{\alpha}(x)\right)=\mathscr{D}^{\alpha}\left(U_{u_{\phi}^{\alpha}}, x\right) .
$$

But, since $U_{u_{\phi}^{\alpha}} \in \mathcal{U}$ and $\mathscr{g}_{D}^{\alpha}(x)=\inf _{U \in \mathcal{U}} \mathscr{D}^{\alpha}(U, x)$, it is clear that $\mathscr{D}^{\alpha}\left(U_{u_{\phi}^{\alpha}}, x\right) \geq \mathscr{g}_{D}^{\alpha}(x)$, so (A.19) yields (A.16).

The next proposition gives a characterization of $\operatorname{g}_{D}^{\alpha}(x)$ in terms of $G$.

Proposition A.3. For $\alpha>0$ and $u_{\phi}^{\alpha}$ as in Lemma A.1, define the sequence $\left(s_{m}^{\alpha}(x)\right)_{m \in \mathbb{N}}$ for $x \in E$ by $s_{0}^{\alpha}(x)=0$ and $s_{m+1}^{\alpha}(x)=L_{\alpha} f\left(x, u_{\phi}^{\alpha}(x)\right)+H_{\alpha} r\left(x, u_{\phi}^{\alpha}(x)\right)+G_{\alpha} s_{m}^{\alpha}\left(x, u_{\phi}^{\alpha}(x)\right)$. Then

$$
g_{D}^{\alpha}(x)=\lim _{m \rightarrow \infty} \sum_{k=0}^{m} G^{k}\left(L\left(f-\alpha s_{m+1-k}^{\alpha}\right)+H r\right)\left(x, u_{\phi}^{\alpha}(x)\right) .
$$

Proof. By definition, for all $m \in \mathbb{N}, s_{m}^{\alpha} \in \mathbb{M}(E)$ and $s_{m+1}^{\alpha}(x)=\sum_{k=0}^{m} G_{\alpha}^{k}\left(L_{\alpha} f+H_{\alpha} r\right) \times$ $\left(x, u_{\phi}^{\alpha}(x)\right)$, and, clearly, from Lemma A.1, we have $s_{m}^{\alpha} \uparrow g_{D}^{\alpha}$ as $m \uparrow \infty$. Applying Lemma 9.2 
of [2], it can be shown that $s_{m}^{\alpha} \in \mathbb{M}^{a c}(E)$ and, for all $x \in E$ and $t \in\left[0, t_{*}(x)\right)$,

$$
\begin{aligned}
& s_{m+1}^{\alpha}(x)=\int_{0}^{t} \exp \left\{-\alpha s-\int_{0}^{s} \lambda\left(\phi(x, \theta), u^{\alpha}(\phi(x, \theta))\right) \mathrm{d} \theta\right\} \\
& \times[ {\left[f\left(\phi(x, s), u^{\alpha}(\phi(x, s))\right)\right.} \\
&\left.+\lambda\left(\phi(x, s), u^{\alpha}(\phi(x, s))\right) Q s_{m}^{\alpha}\left(\phi(x, s), u^{\alpha}(\phi(x, s))\right)\right] \mathrm{d} s \\
&+ \exp \left\{-\alpha t-\int_{0}^{t} \lambda\left(\phi(x, s), u^{\alpha}(\phi(x, s))\right) \mathrm{d} s\right\} s_{m+1}^{\alpha}(\phi(x, t)),
\end{aligned}
$$

implying that

$$
\begin{aligned}
X s_{m+1}^{\alpha}(x)-\left[\alpha+\lambda\left(x, u^{\alpha}(x)\right)\right] s_{m+1}^{\alpha}(x)+f(x, & \left.u^{\alpha}(x)\right) \\
& +\lambda\left(x, u^{\alpha}(x)\right) Q s_{m}^{\alpha}\left(x, u^{\alpha}(x)\right)=0 .
\end{aligned}
$$

Consider the case in which $t_{*}(x)<\infty$. Since $s_{m+1}^{\alpha} \in \mathbb{M}^{a c}(E)$, this yields

$$
\begin{aligned}
s_{m+1}^{\alpha}(x)= & L_{\alpha} f\left(x, u_{\phi}^{\alpha}(x)\right) \\
& +\exp \left\{-\alpha t_{*}(x)-\int_{0}^{t_{*}(x)} \lambda\left(\phi(x, s), u^{\alpha}(\phi(x, s))\right) \mathrm{d} s\right\} s_{m+1}^{\alpha}\left(\phi\left(x, t_{*}(x)\right)\right) \\
& +\int_{0}^{t_{*}(x)} \exp \left\{-\alpha s-\int_{0}^{s} \lambda\left(\phi(x, \theta), u^{\alpha}(\phi(x, \theta))\right) \mathrm{d} \theta\right\} \\
& \quad \times \lambda\left(\phi(x, s), u^{\alpha}(\phi(x, s))\right) Q s_{m}^{\alpha}\left(\phi(x, s), u^{\alpha}(\phi(x, s))\right) \mathrm{d} s .
\end{aligned}
$$

From Assumption 3.3, we have $\exp \left\{-\int_{0}^{t_{*}(x)} \lambda\left(\phi(x, s), u^{\alpha}(\phi(x, s))\right) \mathrm{d} s\right\}>0$. Therefore, combining the definition of $s_{m}^{\alpha}(x)$ and (A.22), we obtain

$$
\begin{aligned}
s_{m+1}^{\alpha}\left(\phi\left(x, t_{*}(x)\right)\right)= & Q s_{m}^{\alpha}\left(\phi\left(x, t_{*}(x)\right), u\left(\phi\left(x, t_{*}(x)\right)\right)\right) \\
& +r\left(\phi\left(x, t_{*}(x)\right), u\left(\phi\left(x, t_{*}(x)\right)\right)\right) .
\end{aligned}
$$

Using Proposition A.2, we obtain, from (A.21) and (A.23),

$$
s_{m+1}^{\alpha}(x)=L\left(f-\alpha s_{m+1}^{\alpha}\right)\left(x, u_{\phi}^{\alpha}(x)\right)+H r\left(x, u_{\phi}^{\alpha}(x)\right)+G s_{m}^{\alpha}\left(x, u_{\phi}^{\alpha}(x)\right) .
$$

Iterations of (A.24) over $m$ yields (A.20).

Before showing that the mapping defined by $g_{D}^{\alpha}(\cdot)-g_{D}^{\alpha}(y)$ for $y$ fixed in $E$ belongs to $\mathbb{B}_{g}(E)$, we need to prove that the mapping $L\left(f-\alpha s_{m+1}^{\alpha}\right)\left(\cdot, u_{\phi}^{\alpha}(\cdot)\right)+\operatorname{Hr}\left(\cdot, u_{\phi}^{\alpha}(\cdot)\right)$ belongs to $\mathbb{B}_{g}(E)$.

Lemma A.2. Define $M^{\prime}=M(1+b / c)\left(1+b K_{\lambda}\right) / c$. For $\alpha>0, u_{\phi}^{\alpha}$ as in Lemma A.1, $s_{m}^{\alpha}$ as in Lemma A.3, and $x \in E$, we have

$$
\left|L\left(f-\alpha s_{m+1}^{\alpha}\right)\left(x, u_{\phi}^{\alpha}(x)\right)+H r\left(x, u_{\phi}^{\alpha}(x)\right)\right| \leq M^{\prime} g(x) .
$$

Proof. Note that

$$
\begin{aligned}
-\alpha L s_{m+1}^{\alpha}\left(x, u_{\phi}^{\alpha}(x)\right) & \leq L\left(f-\alpha s_{m+1}^{\alpha}\right)\left(x, u_{\phi}^{\alpha}(x)\right)+H r\left(x, u_{\phi}^{\alpha}(x)\right) \\
& \leq L f\left(x, u_{\phi}^{\alpha}(x)\right)+H r\left(x, u_{\phi}^{\alpha}(x)\right) .
\end{aligned}
$$


Considering $q_{m}^{\alpha}=s_{m}^{\alpha}$ in Theorem A.1 and recalling that $g \geq 1$, we obtain, from (A.10),

$$
s_{m}^{\alpha}(x) \leq \frac{M}{c+\alpha} g(x)+\frac{M b}{c \alpha} \leq \frac{M(1+b / c)}{\alpha} g(x) .
$$

Therefore, from (A.26) we have $\alpha s_{m}^{\alpha} \leq M(1+b / c) g$ and, thus, from (A.12),

$$
\alpha L s_{m+1}^{\alpha}\left(x, u_{\phi}^{\alpha}(x)\right) \leq \frac{M(1+b / c)\left(1+b K_{\lambda}\right)}{c} g(x) .
$$

By combining (A.11), (A.25), and (A.27), the result follows.

Proof of Theorem 4.2. From Assumption 3.2 and Lemma A.2 we obtain, for all $x \in E$,

$$
\left|G^{k}\left(L\left(f-\alpha s_{m+1-k}^{\alpha}\right)+H r\right)\left(x, u_{\phi}^{\alpha}(x)\right)-\pi_{u^{\alpha}}\left(L\left(f-\alpha s_{m+1-k}^{\alpha}\right)+H r\right)\right| \leq a M^{\prime} \kappa^{k} g(x) .
$$

Consequently,

$$
\begin{aligned}
& \left|\sum_{k=0}^{m} G^{k}\left(L\left(f-\alpha s_{m+1-k}^{\alpha}\right)+H r\right)\left(x, u_{\phi}^{\alpha}(x)\right)-G^{k}\left(L\left(f-\alpha s_{m+1-k}^{\alpha}\right)+H r\right)\left(y, u_{\phi}^{\alpha}(y)\right)\right| \\
& \quad \leq a M^{\prime}(g(x)+g(y)) \frac{1-\kappa^{m+1}}{1-\kappa} .
\end{aligned}
$$

Taking the limit as $m \uparrow \infty$ in the previous equation and recalling that $g \geq 1$ we obtain the desired result from Proposition A.3.

\section{A.3. Existence of an ordinary feedback measurable selector}

The main goal of this subsection is to show that, for any function $h \in \mathbb{B}_{g}(E)$, the onestage optimization operators $\mathcal{R}_{\alpha}(\rho, h)(x)$ and $\mathcal{T}_{\alpha}(\rho, h)(x)$ are equal, and that there exists an ordinary feedback measurable selector for the one-stage optimization problems associated to these operators (see Theorem A.2, below). This theorem is an extension of a result obtained in [2] for the case in which the functions under consideration are not necessarily bounded below, as supposed in [2], but, instead, belong to $\mathbb{B}_{g}(E)$. It must be pointed out that this extension is far from trivial to obtain.

The next two technical lemmas will be used to derive Theorem A.2.

Lemma A.3. Let $\alpha \geq 0, \rho \in \mathbb{R}_{+}$, and $h \in \mathbb{B}_{g}(E)$, and set $w=\mathcal{R}_{\alpha}(\rho, h)$. Then there exists $\hat{\Theta} \in \delta_{\mathbb{V} r}$ such that

$$
w(x)=-\rho \mathcal{L}_{\alpha}(x, \hat{\Theta}(x))+L_{\alpha} f(x, \hat{\Theta}(x))+H_{\alpha} r(x, \hat{\Theta}(x))+G_{\alpha} h(x, \hat{\Theta}(x)) .
$$

Moreover, $w \in \mathbb{M}^{a c}(E)$, satisfying, for all $x \in E$ and $t \in\left[0, t_{*}(x)\right)$,

$$
\begin{aligned}
w(x)= & \inf _{\mu \in \mathcal{V}^{r}(x)}\left\{\int_{0}^{t} \mathrm{e}^{-\alpha s-\Lambda^{\mu}(x, s)}[-\rho+f(\phi(x, s), \mu(s))+\lambda Q h(\phi(x, s), \mu(s))] \mathrm{d} s\right. \\
& \left.\quad+\mathrm{e}^{-\alpha t-\Lambda^{\mu}(x, t)} w(\phi(x, t))\right\} \\
= & \int_{0}^{t} \mathrm{e}^{-\alpha s-\Lambda^{\hat{\mu}(x)}(x, s)}[-\rho+f(\phi(x, s), \hat{\mu}(x, s))+\lambda Q h(\phi(x, s), \hat{\mu}(x, s))] \mathrm{d} s \\
& +\mathrm{e}^{-\alpha t-\Lambda^{\hat{\mu}(x)}(x, t)} w(\phi(x, t)),
\end{aligned}
$$

where $\hat{\Theta}(x)=\left(\hat{\mu}(x), \hat{\mu}_{\partial}(x)\right)$. 
Proof. From Corollary A.1, it follows that the mapping $V$ defined on $\mathcal{K}$ by

$$
V(x, \Theta)=-\rho \mathcal{L}_{\alpha}(x, \Theta)+L_{\alpha} f(x, \Theta)+H_{\alpha} r(x, \Theta)+G_{\alpha} h(x, \Theta)
$$

takes values in $\mathbb{R}$. Moreover, from Assumption 2.5 and Proposition 3.4 of [2], it follows that $V$ is measurable. Furthermore, by using Corollary 5.8 of [2], it follows that, for all $x \in E$, $V(x, \cdot)$ is lower semicontinuous on $\mathbb{V}^{r}(x)$. Recalling that $\mathbb{V}^{r}(x)$ is a compact subset of $\mathbb{V}^{r}$ and by using Proposition D.5 of [10], we find that there exists $\hat{\Theta} \in \diamond_{\mathbb{V}^{r}}$ such that (A.28) is satisfied. The rest of the proof is similar to the proof of Proposition 4.2 of [2] and is therefore omitted.

Lemma A.4. Let $\alpha \geq 0, \rho \in \mathbb{R}_{+}$, and $h \in \mathbb{B}_{g}(E)$. Then, for all $x \in E$,

$$
\mathcal{R}_{\alpha}(\rho, h)(x) \geq-\left(\rho+b\|h\|_{g}\right) K_{\lambda}-\|h\|_{g} g(x),
$$

and, for all $x \in E$ such that $t_{*}(x)=\infty$ and $\Theta=\left(\mu, \mu_{\partial}\right) \in \mathbb{V}^{r}(x)$,

$$
\begin{aligned}
-\rho \mathcal{L}_{\alpha}(x, \Theta)+L_{\alpha} f(x, \Theta)+H_{\alpha} r(x, \Theta)+G_{\alpha} h(x, \Theta) \\
\quad=\lim _{t \rightarrow+\infty} \int_{0}^{t} \mathrm{e}^{-\alpha s-\Lambda^{\mu}(x, s)}[-\rho+f(\phi(x, s), \mu(s))+\lambda Q h(\phi(x, s), \mu(s))] \mathrm{d} s .
\end{aligned}
$$

Proof. From (A.8) we have

$$
G_{\alpha} g(x, \Theta) \leq b K_{\lambda}+g(x)
$$

for all $x \in E$ and $\Theta \in \mathbb{V}^{r}$. Consequently, using (A.28) and the fact that $f \geq 0$ and $r \geq 0$, it follows that

$$
\mathcal{R}_{\alpha}(\rho, h)(x) \geq-\rho \mathcal{L}_{\alpha}(x, \hat{\Theta}(x))+G_{\alpha} h(x, \hat{\Theta}(x)) \geq-\left(\rho+b\|h\|_{g}\right) K_{\lambda}-\|h\|_{g} g(x),
$$

showing the first part of the result. From Assumption 3.6(a), (b), and (e), we have

$$
\lim _{t \rightarrow+\infty} \int_{0}^{t} \mathrm{e}^{-\alpha s-\Lambda^{\mu}(x, s)}[-\rho+f(\phi(x, s), \mu(s))] \mathrm{d} s
$$

exists in $\mathbb{R}$, and from (A.33),

$$
\lim _{t \rightarrow+\infty} \int_{0}^{t} \mathrm{e}^{-\alpha s-\Lambda^{\mu}(x, s)} \lambda Q g(\phi(x, s), \mu(s)) \mathrm{d} s
$$

exists in $\mathbb{R}$. Using the fact that $h \in \mathbb{B}_{g}(E)$, it follows that the limit on the right-hand side of (A.32) exists. Finally, from Remark 3.1(i) we obtain the last part of the result.

The next result shows that, for any function $h \in \mathbb{B}_{g}(E)$, the one-stage optimization operators $\mathcal{R}_{\alpha}(\rho, h)(x)$ and $\mathcal{T}_{\alpha}(\rho, h)(x)$ are equal, and that there exists an ordinary feedback measurable selector for the one-stage optimization problems associated to these operators.

Theorem A.2. Let $\alpha \geq 0, \rho \in \mathbb{R}_{+}$, and $h \in \mathbb{B}_{g}(E)$, and set $w=\mathcal{R}_{\alpha}(\rho, h)$. Then $w \in \mathbb{M}^{a c}(E)$ and the ordinary feedback measurable selector $\hat{u}_{\phi}(w, h) \in \$_{\mathbb{V}}$ (see Definition 3.1(D2)) satisfies the following one-stage optimization problems:

$$
\begin{aligned}
\mathcal{R}_{\alpha}(\rho, h)(x)= & \mathcal{T}_{\alpha}(\rho, h)(x) \\
= & -\rho \mathcal{L}_{\alpha}\left(x, \hat{u}_{\phi}(w, h)(x)\right)+L_{\alpha} f\left(x, \hat{u}_{\phi}(w, h)(x)\right)+H_{\alpha} r\left(x, \hat{u}_{\phi}(w, h)(x)\right) \\
& +G_{\alpha} h\left(x, \hat{u}_{\phi}(w, h)(x)\right) .
\end{aligned}
$$


Proof. According to Lemma A.3, there exists $\hat{\Theta} \in \delta_{\mathbb{V} r}$ such that, for all $x \in E$ and $t \in$ $\left[0, t_{*}(x)\right)$, we have

$$
\begin{aligned}
& \mathrm{e}^{-\alpha t-\Lambda^{\hat{\mu}(x)}(x, t)} w(\phi(x, t))-w(x) \\
& \quad=\int_{0}^{t} \mathrm{e}^{-\alpha s-\Lambda^{\hat{\mu}(x)}(x, s)}[\rho-f(\phi(x, s), \hat{\mu}(x, s))-\lambda Q h(\phi(x, s), \hat{\mu}(x, s))] \mathrm{d} s,
\end{aligned}
$$

where $\hat{\Theta}(x)=\left(\hat{\mu}(x), \hat{\mu}_{\partial}(x)\right)$. Since $w \in \mathbb{M}^{a c}(E)$, we obtain, from (A.34),

$$
\begin{aligned}
& -\mathcal{X} w(\phi(x, t))+\alpha w(\phi(x, t)) \\
& \quad \geq \inf _{\mu \in \mathcal{P}(\mathbb{U}(\phi(x, t)))}\{f(\phi(x, t), \mu)-\lambda(\phi(x, t), \mu) w(\phi(x, t))+\lambda Q h(\phi(x, t), \mu)\}-\rho .
\end{aligned}
$$

However, note that

$$
\begin{aligned}
& \inf _{\mu \in \mathcal{P}(\mathbb{U}(\phi(x, t)))}\{f(\phi(x, t), \mu)-\lambda(\phi(x, t), \mu) w(\phi(x, t))+\lambda Q h(\phi(x, t), \mu)\}-\rho \\
& \quad=\inf _{a \in \mathbb{U}(\phi(x, t))}\{f(\phi(x, t), a)-\lambda(\phi(x, t), a)[w(\phi(x, t))-Q h(\phi(x, t), a)]\}-\rho .
\end{aligned}
$$

Consequently, by considering the measurable selector $\bar{u} \in \delta_{\mathbb{U}}$ given by $\bar{u}=\hat{u}(w, h)$ (see Definition 3.1(D1)), we have

$$
\begin{aligned}
-X w(\phi(x, t))+\alpha w(\phi(x, t)) & \\
= & -\rho+f(\phi(x, t), \bar{u}(\phi(x, t))) \\
& -\lambda(\phi(x, t), \bar{u}(\phi(x, t)))[w(\phi(x, t))-Q h(\phi(x, t), \bar{u}(\phi(x, t)))]
\end{aligned}
$$

$\eta$-almost surely on $\left[0, t_{*}(x)\right)$, otherwise this would be in contradiction with (A.29). Consequently, for all $t \in\left[0, t_{*}(x)\right)$, it follows that

$$
\begin{aligned}
w(x)=\mathrm{e}^{-(\alpha t+\bar{\Lambda}(x, t))} w(\phi(x, t)) & \\
+\int_{0}^{t} \mathrm{e}^{-(\alpha s+\bar{\Lambda}(x, s))}[ & f(\phi(x, s), \bar{u}(\phi(x, s))) \\
& +\lambda(\phi(x, s), \bar{u}(\phi(x, s))) Q h(\phi(x, s), \bar{u}(\phi(x, s)))-\rho] \mathrm{d} s,
\end{aligned}
$$

where $\bar{\Lambda}(x, t)=\int_{0}^{t} \lambda(\phi(x, s), \bar{u}(\phi(x, s))) \mathrm{d} s$. First consider the case in which $t_{*}(x)<\infty$. We find, by taking the limit as $t$ tends to $t_{*}(x)$ in the previous equation, that the ordinary feedback measurable selector $\hat{u}_{\phi}(w, h) \in \$_{\mathbb{V}}($ see Definition 3.1(D2)) satisfies

$$
\begin{aligned}
w(x)= & \mathrm{e}^{-\left(\alpha t_{*}(x)+\bar{\Lambda}\left(x, t_{*}(x)\right)\right)} w\left(\phi\left(x, t_{*}(x)\right)\right)-\rho \mathcal{L}_{\alpha}\left(x, \hat{u}_{\phi}(w, h)(x)\right)+L_{\alpha} f\left(x, \hat{u}_{\phi}(w, h)(x)\right) \\
& +\int_{0}^{t_{*}(x)} \mathrm{e}^{-(\alpha s+\bar{\Lambda}(x, s))} \lambda(\phi(x, s), \bar{u}(\phi(x, s))) Q h(\phi(x, s), \bar{u}(\phi(x, s))) \mathrm{d} s .
\end{aligned}
$$

Define the control $\Theta(x)$ by $(\hat{\mu}(x), \mu)$ for $\mu \in \mathcal{P}\left(\mathbb{U}\left(\phi\left(x, t_{*}(x)\right)\right)\right)$. Therefore, we have

$$
\begin{aligned}
w(x) \leq & -\rho \mathcal{L}_{\alpha}(x, \hat{\Theta}(x))+L_{\alpha} f(x, \hat{\Theta}(x)) \\
& +\int_{0}^{t_{*}(x)} \mathrm{e}^{-\alpha s-\Lambda^{\hat{\mu}(x)}(x, s)} \lambda Q h(\phi(x, s), \hat{\mu}(x, s)) \mathrm{d} s \\
& +\mathrm{e}^{-\alpha t_{*}(x)-\Lambda^{\hat{\mu}(x)}\left(x, t_{*}(x)\right)}\left[\operatorname{Qh}\left(\phi\left(x, t_{*}(x)\right), \mu\right)+r\left(\phi\left(x, t_{*}(x)\right), \mu\right)\right] .
\end{aligned}
$$


From (A.30) and since $w \in \mathbb{M}^{a c}(E)$, we have

$$
\begin{aligned}
w(x)= & -\rho \mathcal{L}_{\alpha}(x, \hat{\Theta}(x))+L_{\alpha} f(x, \hat{\Theta}(x)) \\
& +\int_{0}^{t_{*}(x)} \mathrm{e}^{-\alpha s-\Lambda^{\hat{\mu}(x)}(x, s)} \lambda Q h(\phi(x, s), \hat{\mu}(x, s)) \mathrm{d} s \\
& +\mathrm{e}^{-\alpha t_{*}(x)-\Lambda^{\hat{\mu}(x)}\left(x, t_{*}(x)\right)} w\left(\phi\left(x, t_{*}(x)\right)\right) .
\end{aligned}
$$

By Assumption 3.3, we have $\mathrm{e}^{-\Lambda^{\hat{\mu}(x)}\left(x, t_{*}(x)\right)}>0$. Therefore, combining (A.37) and (A.38), we obtain, for all $x \in E$ and $\mu \in \mathcal{P}\left(\mathbb{U}\left(\phi\left(x, t_{*}(x)\right)\right)\right)$,

$$
w\left(\phi\left(x, t_{*}(x)\right)\right) \leq Q h\left(\phi\left(x, t_{*}(x)\right), \mu\right)+r\left(\phi\left(x, t_{*}(x)\right), \mu\right) .
$$

Clearly, by using (A.28), it can be claimed that the previous inequality becomes an equality for $\mu=\hat{\mu}_{\partial}(x)$, implying that

$$
\begin{aligned}
w\left(\phi\left(x, t_{*}(x)\right)\right) & =\inf _{\mu \in \mathcal{P}\left(\mathbb{U}\left(\phi\left(x, t_{*}(x)\right)\right)\right)}\left\{r\left(\phi\left(x, t_{*}(x)\right), \mu\right)+Q h\left(\phi\left(x, t_{*}(x)\right), \mu\right)\right\} \\
& =\inf _{a \in \mathbb{U}\left(\phi\left(x, t_{*}(x)\right)\right)}\left\{r\left(\phi\left(x, t_{*}(x)\right), a\right)+Q h\left(\phi\left(x, t_{*}(x)\right), a\right)\right\} .
\end{aligned}
$$

Consequently, we have

$$
w\left(\phi\left(x, t_{*}(x)\right)\right)=r\left(\phi\left(x, t_{*}(x)\right), \bar{u}\left(\phi\left(x, t_{*}(x)\right)\right)\right)+Q h\left(\phi\left(x, t_{*}(x)\right), \bar{u}\left(\phi\left(x, t_{*}(x)\right)\right)\right) .
$$

Combining (A.36) and (A.39), it follows that

$$
\begin{aligned}
w(x)= & -\rho \mathcal{L}_{\alpha}\left(x, \hat{u}_{\phi}(w, h)(x)\right)+L_{\alpha} f\left(x, \hat{u}_{\phi}(w, h)(x)\right)+H_{\alpha} r\left(x, \hat{u}_{\phi}(w, h)(x)\right) \\
& +G_{\alpha} h\left(x, \hat{u}_{\phi}(w, h)(x)\right) .
\end{aligned}
$$

Now consider the case in which $t_{*}(x)=\infty$. Using (A.31) and (A.35), we obtain

$$
\begin{aligned}
w(x) \geq- & \mathrm{e}^{-(\alpha t+\bar{\Lambda}(x, t))}\left[\left(\rho+b\|h\|_{g}\right) K_{\lambda}+\|h\|_{g} g(\phi(x, t))\right] \\
+ & \quad \int_{0}^{t} \mathrm{e}^{-(\alpha s+\bar{\Lambda}(x, s))}[ \\
& f(\phi(x, s), \bar{u}(\phi(x, s))) \\
& +\lambda(\phi(x, s), \bar{u}(\phi(x, s))) Q h(\phi(x, s), \bar{u}(\phi(x, s)))-\rho] \mathrm{d} s .
\end{aligned}
$$

However, from Assumption 3.6(a) and (d) we obtain

$$
\lim _{t \rightarrow+\infty} \mathrm{e}^{-(\alpha t+\bar{\Lambda}(x, t))}\left[\left(\rho+b\|h\|_{g}\right) K_{\lambda}+\|h\|_{g} g(\phi(x, t))\right]=0 .
$$

Consequently, combining (A.32), (A.40), and (A.41), the ordinary feedback measurable selector $\hat{u}_{\phi}(w, h) \in \delta_{\mathbb{V}}$ satisfies

$$
\begin{aligned}
w(x) \geq & -\rho+\mathcal{L}_{\alpha}\left(x, \hat{u}_{\phi}(w, h)(x)\right)+L_{\alpha} f\left(x, \hat{u}_{\phi}(w, h)(x)\right)+H_{\alpha} r\left(x, \hat{u}_{\phi}(w, h)(x)\right) \\
& +G_{\alpha} h\left(x, \hat{u}_{\phi}(w, h)(x)\right) .
\end{aligned}
$$

Using (A.29), it follows that the inequality in the previous equation is in fact an equality.

In conclusion, since $\mathbb{V}(x) \subset \mathbb{V}^{r}(x)$, it follows that $\mathcal{R}_{\alpha}(\rho, h)(x) \leq \mathcal{T}_{\alpha}(\rho, h)(x)$. However, we have shown that $\hat{u}_{\phi}(w, h) \in \mathcal{S}_{\mathbb{V}}$ satisfies

$$
\begin{aligned}
\mathcal{R}_{\alpha}(\rho, h)(x)= & -\rho \mathcal{L}_{\alpha}\left(x, \hat{u}_{\phi}(w, h)(x)\right)+L_{\alpha} f\left(x, \hat{u}_{\phi}(w, h)(x)\right)+H_{\alpha} r\left(x, \hat{u}_{\phi}(w, h)(x)\right) \\
& +G_{\alpha} h\left(x, \hat{u}_{\phi}(w, h)(x)\right),
\end{aligned}
$$

which is the desired result. 


\section{Acknowledgements}

OLVC received financial support from CNPq (Brazilian National Research Council), grant number 304866/03-2, and FAPESP (Research Council of the State of São Paulo), grant number 03/06736-7. FD was partially supported by the ARPEGE program of the French National Agency of Research (ANR), project 'FAUTOCOES', grant number ANR-09-SEGI-004. The authors would like to express their gratitude to the Editor and anonymous referee for their suggestions and helpful comments.

\section{References}

[1] Costa, O. L. V. And Dufour, F. (2007). Relaxed long-run average continuous control of piecewise deterministic Markov processes. In Proc. European Control Conf., Kos, Greece, 5052-5059.

[2] Costa, O. L. V. And Dufour, F. (2008). Average continuous control of piecewise deterministic Markov processes. Submitted. Available at http://arxiv.org/abs/0809.0477.

[3] Costa, O. L. V. And Dufour, F. (2008). Stability and ergodicity of piecewise deterministic Markov processes. SIAM J. Control Optimization 47, 1053-1077.

[4] Davis, M. H. A. (1984). Piecewise-deterministic Markov processes: a general class of non-diffusion stochastic models. J. R. Statist. Soc. B 46, 353-388.

[5] Davis, M. H. A. (1993). Markov Models and Optimization. Chapman and Hall, London.

[6] Davis, M. H. A., Dempster, M. A. H., Sethi, S. P. and Vermes, D. (1987). Optimal capacity expansion under uncertainty. Adv. Appl. Prob. 19, 156-176.

[7] Dufour, F. and Costa, O. L. V. (1999). Stability of piecewise-deterministic Markov processes. SIAM J. Control Optimization 37, 1483-1502.

[8] Guo, X. AND RIEdER, U. (2006). Average optimality for continuous-time Markov decision processes in Polish spaces. Ann. Appl. Prob. 16, 730-756.

[9] Guo, X. And ZHu, Q. (2006). Average optimality for Markov decision processes in Borel spaces: a new condition and approach. J. Appl. Prob. 43, 318-334.

[10] Hernández-Lerma, O. and Lasserre, J. B. (1996). Discrete-Time Markov Control Processes (Appl. Math. 30). Springer, New York.

[11] Hernández-Lerma, O. and Lasserre, J. B. (1999). Further Topics on Discrete-Time Markov Control Processes (Appl. Math. 42). Springer, New York.

[12] Luss, H. (1982). Operations research and capacity expansion problems: a survey. Operat. Res. 30, $907-947$.

[13] Meyn, S. ANd Tweedie, R. (1992). Stability of Markovian processes. I. Criteria for discrete-time chains. Adv. Appl. Prob. 24, 542-574.

[14] Meyn, S. P. And Tweedie, R. L. (1993). Markov Chains and Stochastic Stability. Springer, Berlin.

[15] Widder, D. V. (1941). The Laplace Transform (Princeton Math. Ser. v. 6). Princeton University Press.

[16] ZHU, Q. (2008). Average optimality for continuous-time Markov decision processes with a policy iteration approach. J. Math. Anal. Appl. 339, 691-704. 